\title{
A zebrafish model of tauopathy allows in vivo imaging of neuronal cell death and drug evaluation
}

\author{
Dominik Paquet, ${ }^{1,2}$ Ratan Bhat, ${ }^{3}$ Astrid Sydow, ${ }^{4}$ Eva-Maria Mandelkow, ${ }^{4}$ \\ Stefan Berg, ${ }^{3}$ Sven Hellberg, ${ }^{3}$ Johanna Fälting, ${ }^{3}$ Martin Distel, ${ }^{5}$ \\ Reinhard W. Köster, ${ }^{5}$ Bettina Schmid,, ${ }^{1,2}$ and Christian Haass ${ }^{1,2}$ \\ ${ }^{1}$ Deutsches Zentrum für Neurodegenerative Erkrankungen (DZNE) and ${ }^{2}$ Adolf-Butenandt-Institute, \\ Biochemistry, Ludwig-Maximilians-University, Munich, Germany. \\ ${ }^{3}$ AstraZeneca Research \& Development, Södertälje, Sweden. ${ }^{4}$ Max-Planck Unit for Structural Molecular Biology at DESY, Hamburg, Germany. \\ ${ }^{5}$ Helmholtz Zentrum München, Institute of Developmental Genetics, Neuherberg, Germany.
}

\begin{abstract}
Our aging society is confronted with a dramatic increase of patients suffering from tauopathies, which include Alzheimer disease and certain frontotemporal dementias. These disorders are characterized by typical neuropathological lesions including hyperphosphorylation and subsequent aggregation of TAU protein and neuronal cell death. Currently, no mechanism-based cures are available. We generated fluorescently labeled TAU transgenic zebrafish, which rapidly recapitulated key pathological features of tauopathies, including phosphorylation and conformational changes of human TAU protein, tangle formation, neuronal and behavioral disturbances, and cell death. Due to their optical transparency and small size, zebrafish larvae are well suited for both in vivo imaging and drug development. TAU-induced neuronal cell death was imaged by time-lapse microscopy in vivo. Furthermore, we used this zebrafish model to identify compounds targeting the TAU kinase glycogen synthase kinase $3 \beta$ (GSK3 $\beta$ ). We identified a newly developed highly active GSK3 $\beta$ inhibitor, AR-534, by rational drug design. AR-534 reduced TAU phosphorylation in TAU transgenic zebrafish. This transgenic zebrafish model may become a valuable tool for further studies of the neuropathology of dementia.
\end{abstract}

\section{Introduction}

Neurodegenerative diseases are the most frequent cause of dementia in our aging society. For these disorders, which include Alzheimer disease (AD) and frontotemporal dementia (FTD), disease-modifying treatments represent a highly unmet medical need. AD and FTD are characterized by posttranslationally modified amyloidogenic proteins, which form neurotoxic oligomers and are finally deposited as insoluble aggregates (1). Examples of the proteinaceous building blocks of these deposits are amyloid $\beta$ peptide in $\operatorname{AD}$ and TAU in $\operatorname{AD}$ and $\operatorname{FTD}(2,3)$. The TAU protein is an important target for research and drug development, since its pathological alterations strongly correlate with disease progression in AD and FTD and other neurodegenerative diseases (4) and TAU suppression improves memory function (5). Furthermore, mutations in the TAU-encoding gene microtubuleassociated protein TAU (MAPT) have been found in patients with FTD with Parkinsonism linked to chromosome 17 (FTDP-17) (6). One of the first characteristic modifications of TAU is its pathologic phosphorylation at multiple residues, which are dis-

Conflict of interest: D. Paquet, A. Sydow, E.-M. Mandelkow, M. Distel, R.W. Köster, and B. Schmid declare no conflict of interest. R. Bhat, S. Berg, S. Hellberg, and J. Fälting are employees of AstraZeneca R\&D. C. Haass is a consultant for Elan Pharmaceuticals and receives research support from Boehringer Ingelheim KG.

Nonstandard abbreviations used: $\mathrm{AD}$, Alzheimer disease; $\mathrm{BBB}$, blood-brain barrier; CDK2, cyclin-dependent kinase 2; dN-GSK3 $\beta$, dominant-negative

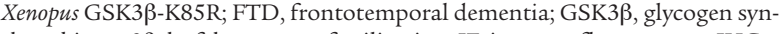
thase kinase $3 \beta$; hpf, hours post fertilization; IF, immunofluorescence; IHC, immunohistochemistry; Ins, insert; MCS, multiple cloning site; UAS, upstream activating sequence; WB, Western blot.

Citation for this article: J. Clin. Invest. 119:1382-1395 (2009). doi:10.1172/JCI37537. tributed over the whole protein $(3,7)$. The initial steps, which lead to TAU phosphorylation and subsequent aggregation, were mainly studied in cell culture but are still poorly understood in vivo. These early stages are difficult to investigate in transgenic mice, since it is not feasible to image cells accurately over longer periods during early disease stages (8). Furthermore, vertebrate in vivo models for rapid high-throughput screening for inhibitors of TAU pathology are not available.

Here, we propose the zebrafish as a small vertebrate model of tauopathies and other neurodegenerative diseases. It offers genetic tractability in combination with a translucent embryo allowing imaging of disease progression at cellular and subcellular levels in the living animal. Furthermore, the potential of using small zebrafish embryos for large-scale drug screening has already been demonstrated (9). Large amounts of small fish, which can be analyzed in a 96-well format, can be rapidly produced. Fish are kept in water, which facilitates uptake of substances from the aqueous medium, allowing for automated compound application. In addition, simple readouts of characteristics such as altered mobility and fluorescence or changes in biochemical composition of proteins can be analyzed on a large scale. Moreover, the endothelial blood-brain barrier (BBB) begins to become functional 3 days after fertilization in zebrafish and has a structure similar to that in higher vertebrates, allowing one to test for bioavailability of drugs in the nervous system (10).

We have optimized the transgenic expression of the human protein TAU-P301L (a mutation genetically linked to FTD; ref. 6) in zebrafish neurons by a newly designed Gal4-upstream activating sequence-based (Gal4/UAS-based) vector system, which also great- 
ly facilitates identification of the transgenic fish by a simultaneously expressed fluorescent reporter. We have further advanced the system to allow introduction of various other genes and promoters to facilitate the generation of different zebrafish disease models. In contrast to previous studies $(11,12)$, we were able to monitor early pathology, including disease-specific hyperphosphorylation and conformational changes of TAU, neuronal and behavioral abnormalities as well as increased neuronal cell death within the first days of embryonic development in stable transgenic zebrafish. The rapid appearance of a pathologic phenotype allows one not only to use the embryos to study disease progression in a transparent vertebrate but also to validate and even screen on a relatively large scale for compounds that modify early phenotypes, such as TAU hyperphosphorylation, in vivo.

Hyperphosphorylation of TAU is believed to be a key initiator of detachment of normal TAU from microtubules and subsequent oligomerization and aggregation. Several kinases, including glycogen synthase kinase $3 \beta$ (GSK3 $\beta$ ), cyclin-dependent kinase 5 (CDK5), ERK2, and microtubule affinity-regulating kinase (MARK), directly phosphorylate TAU and are therefore considered as key therapeutic targets (13). Inhibitors that selectively block these enzymes could therefore be used to slow the progression of disease pathology. Over the last years, selective inhibitors for kinases involved in TAU phosphorylation have been identified, and some of these compounds have even been tested in TAU transgenic mice, where they showed some effects on TAU phosphorylation. The activity of these inhibitors, however, has been hampered by poor water solubility and bioavailability and substantial side effects (13). In addition, large quantities of the compounds are needed, since long-term administration is usually required to observe potential efficacy. Therefore, there is a significant need for technologies that can rapidly and efficiently identify more active and specific compounds with optimized bioavailability in in vivo models that represent disease pathology. We have shown previously that novel substances can be developed by structurebased design, which uses crystal structures of proteins, to model small molecules into the active site of the targeted enzyme (14). This approach can deliver inhibitors with excellent potency and specificity. However, it requires validating the bioavailability and selective inhibition of the target molecule in an in vivo setting. We have now developed several highly specific inhibitors by structurebased design, which bind to the active site of GSK3 $\beta$ and block the enzyme function with high potency in vitro. Two of the best inhibitors were then investigated for their potential efficacy in zebrafish. We demonstrate here for the first time, to our knowledge, that we could reduce the pathologic phosphorylation of the human disease-associated protein TAU in vivo in a transgenic zebrafish model by several published GSK3 $\beta$ inhibitors. Furthermore, we tested 2 newly developed highly selective GSK3 $\beta$ inhibitors with potent in vitro activity and could show with our model that one of them is also very potent in a whole organism in vivo.

Our study not only provides a small vertebrate model of tauopathies and other neurodegenerative diseases, which can be used to monitor progression of pathology in vivo, but also a powerful combination of in silico prediction of compounds with in vivo validation of their bioavailability in a small transgenic vertebrate disease model. Finally, our newly developed zebrafish transgenesis vectors will allow modeling of numerous human disorders that are based on protein misfolding and overexpression, 2 phenomena often connected to each other (15).

\section{Results}

A Gal4/UAS-based bidirectional expression system in zebrafish. The first goal of this study was to generate transgenic zebrafish stably expressing the FTD-associated human protein TAU-P301L (6). Inefficient transgenesis and low protein expression levels have in the past hampered the efficient generation of zebrafish disease models expressing human genes. To overcome these limitations, we have generated vectors that combine several features to increase transgenesis rates and protein levels, facilitate the expression of disease proteins of interest, and allow efficient identification of transgenic lines as well as simultaneous in vivo monitoring of pathology and phenotypes. We cloned 2 constructs, Driver and Responder, based on the medaka Tol2 transposable element (16), which greatly increases the rate of transgenesis (see Methods for details), and integrated the Gal4/UAS expression system (17) into the 2 vectors (Figure 1A). Furthermore, we introduced Gateway recombination sites, which allow rapid introduction of other genes and promoters (18) (see Supplemental Figure 1 for details; supplemental material available online with this article; doi:10.1172/JCI37537DS1). The Driver construct contains the neuronal promoter $\mathrm{HuC}$ (19), controlling the expression of a Gal4-VP16 fusion protein, which in turn efficiently transactivates and amplifies protein expression from a UAS on the Responder construct. To achieve transgene expression in 2 orientations, we flanked the UAS sequence with 2 short minimal promoters. In our constructs, this cassette drives the expression of human TAU-P301L in one direction and the expression of the fluorescent reporter DsRed in the other (Figure 1A). This bidirectional expression allows the identification of TAU-expressing cells in live embryos by concomitant DsRed fluorescence.

Transgenic fish were generated by injecting circular Driver and Responder constructs together with Tol2 transposase mRNA, which is translated into active transposase (a protein not encoded by the zebrafish genome) in embryonic cells to catalyze integration of both constructs into the zebrafish genome for a short period of time (16). Both constructs integrate randomly into a subset of embryonic cells leading to mosaic TAU- and DsRed-expressing embryos. DsRed-positive embryos are raised and outcrossed to wild-type fish. The offspring of founder fish with germ-line transmission can be easily identified, as the embryos express DsRed in mature neurons, making PCR screenings dispensable (Figure 1B). The expression of TAU and DsRed in the transgenic zebrafish fully overlaps, as shown by immunofluorescence (IF) staining using the pan-TAU antibody T46 (20) and DsRed antibodies (Figure 1C).

We raised 76 injected founder fish to sexual maturity and identified 15 (19.7\%) with DsRed-positive offspring. We analyzed 3 generations descending from 1 of the founder fish for genetic inheritance by counting DsRed-negative and -positive embryos and always found about one-fourth of the offspring to be DsRed positive, implying that these embryos carry Driver and Responder constructs (Supplemental Figure 2A). This ratio indicates independent inheritance of both constructs, with single or multiple insertions at 2 different genomic loci. We verified this by analyzing 225 embryos of an F2 outcross by PCR for Driver and Responder genotypes (Supplemental Figure 2B). In addition, the embryos showed stable protein levels and expression domains over 3 generations (Supplemental Figure 2C), indicating stable inheritance and activity of the Gal4/UAS transgene. Finally, we did not observe any morphological alterations in the transgenic fish (data not shown) and also excluded alterations in the function of the zebrafish BBB (for details see Supplemental Figure 3). 
A

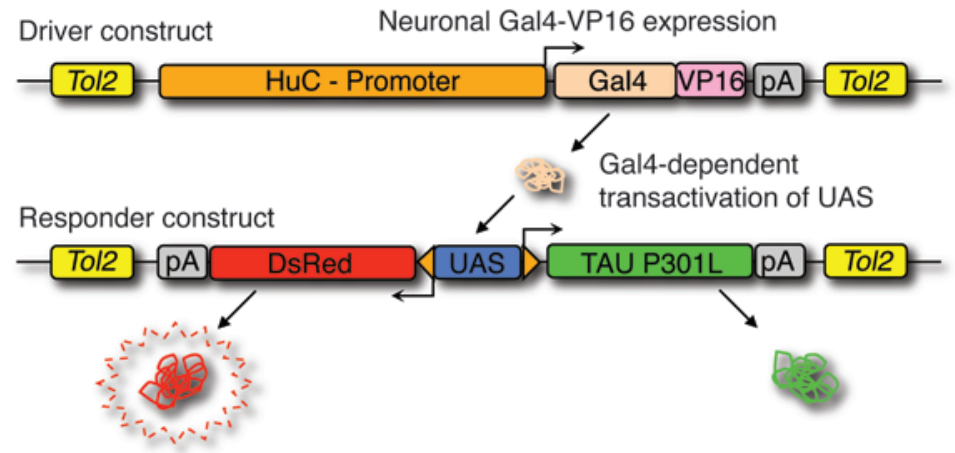

B
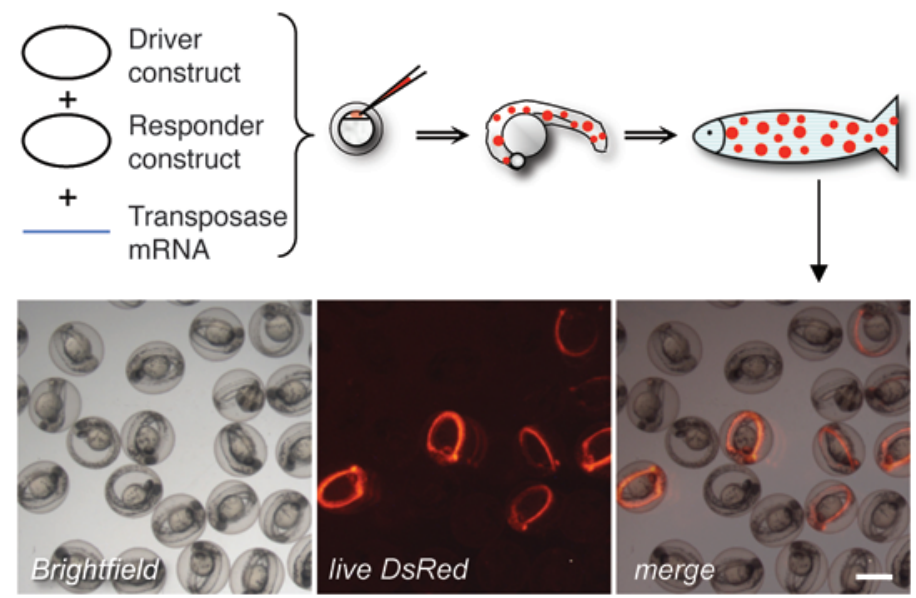

C
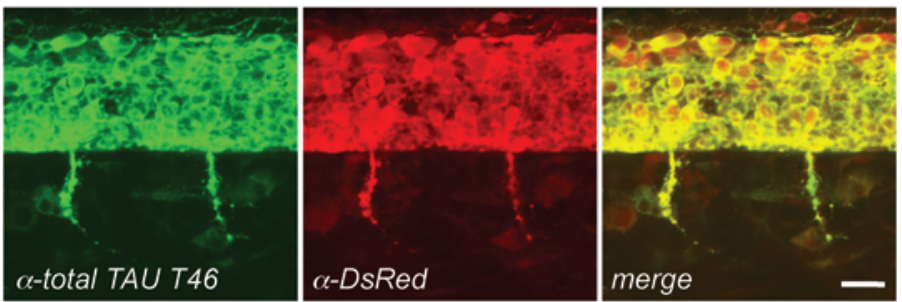

\section{Figure 1}

A Gal4/UAS-based bidirectional expression system in zebrafish. (A) The Driver construct contains the neuronal zebrafish promoter HuC driving the expression of Gal4VP16, which binds to the UAS on the Responder construct. Here, it activates the bidirectional expression of hTAU-P301L and DsRed via the minimal promoters. UAS-dependent gene expression of TAU and DsRed is indicated in living fish by DsRed fluorescence. Driver and Responder constructs are flanked by Tol2 transposon sites. (B) To generate transgenic fish, the Driver and Responder constructs were mixed and injected together with Tol2 mRNA. The mRNA is translated to active transposase, which detects the flanking Tol2 elements and catalyzes random integration into the zebrafish genome in a subset of embryonic cells for a short time period, generating mosaic founder embryos. Mosaic DsRed-positive larvae were raised and outcrossed with wild-type fish. A subset of the offspring will be transgenic and can be easily identified and sorted by DsRed-positive neurons. Scale bar: $1 \mathrm{~mm}$. (C) Double immunostainings for total TAU (T46 antibody) and DsRed of 32-hpf transgenic zebrafish embryos expressing hTAU-P301L and DsRed. Transgenic embryos express both hTAU-P301L and DsRed in spinal cord neurons, showing effective bidirectional expression from the Responder construct. Lateral views of the trunk above the end of the yolk extension, anterior to the left. Scale bar: $20 \mu \mathrm{m}$.
The transgenic zebrafish rapidly develop disease-specific alterations. Phosphorylation of TAU at certain serine and threonine residues serves as a biochemical marker for pathologic alterations in $\operatorname{AD}$ and $\operatorname{FTD}(3,7)$. We therefore determined whether and when the TAU transgenic fish recapitulate this hallmark of disease progression. Strikingly, in embryos that were only 32 hours old, we could already detect positive immunoreactivity in spinal cord neurons with the antibodies AT180 (21), AT270 (21), 12E8 (22), PHF1 (23), 422 (24), and AT8 (25), which specifically detect abnormally phosphorylated residues T231/S235, T181, S262/S356, S396/S404, S422, and S202/T205, respectively (Figure $2 \mathrm{~A})$. These findings were corroborated by immunoblots of lysates from 48-hour-old transgenic and nontransgenic fish. The pan-TAU antibody T46 reveals a broad band around $64 \mathrm{kDa}$, which corresponds to the largest CNS TAU isoform in a mixed state of phosphorylation. Human TAU was also recognized by antibodies against phosphorylated epitopes that are typically elevated in AD (AT180, AT270, 12E8, PHF1, and 422) (Figure 2B).

In addition to pathologic hyperphosphorylation, TAU also changes its conformation during disease progression (26), which finally culminates in aggregation and formation of tangles. The early con- formational changes can be monitored by immunostaining using antibody MC1, which is specific for a pathologic conformation of TAU (26). Strikingly, we already found MC1-positive TAU in neurons of 32-hour-old embryos, again demonstrating rapid occurrence of AD- and FTD-like pathology in our transgenic zebrafish model (Figure 2C). We also determined whether and when these changes to pathologic conformation led to the formation of tangles and therefore analyzed later stages. In 5-week-old fish, which are still quite transparent, we could not only observe the expression of the DsRed transgene in living fish (Figure 2D) but also depict TAU-expressing cells with the pathologic AT8 epitope in immunohistochemical stainings (Figure 2E) and Gallyas silver-positive tangles (Figure 2F) in paraffin sections of spinal cord tissue. To demonstrate specificity of the stainings, we also stained control transgenic lines, which express DsRed only, and nontransgenic siblings. No cross-reactivity in neurons was observed (Figure 2F and Supplemental Figure 4).

Rapid progression of AD/FTD-like late-stage pathology in TAU transgenic fish. We have observed remarkable differences in the amount of TAUexpressing cells, which are stained by early pathology-marking antibodies, such as AT180, AT270 and 12E8, versus late markers, such as 


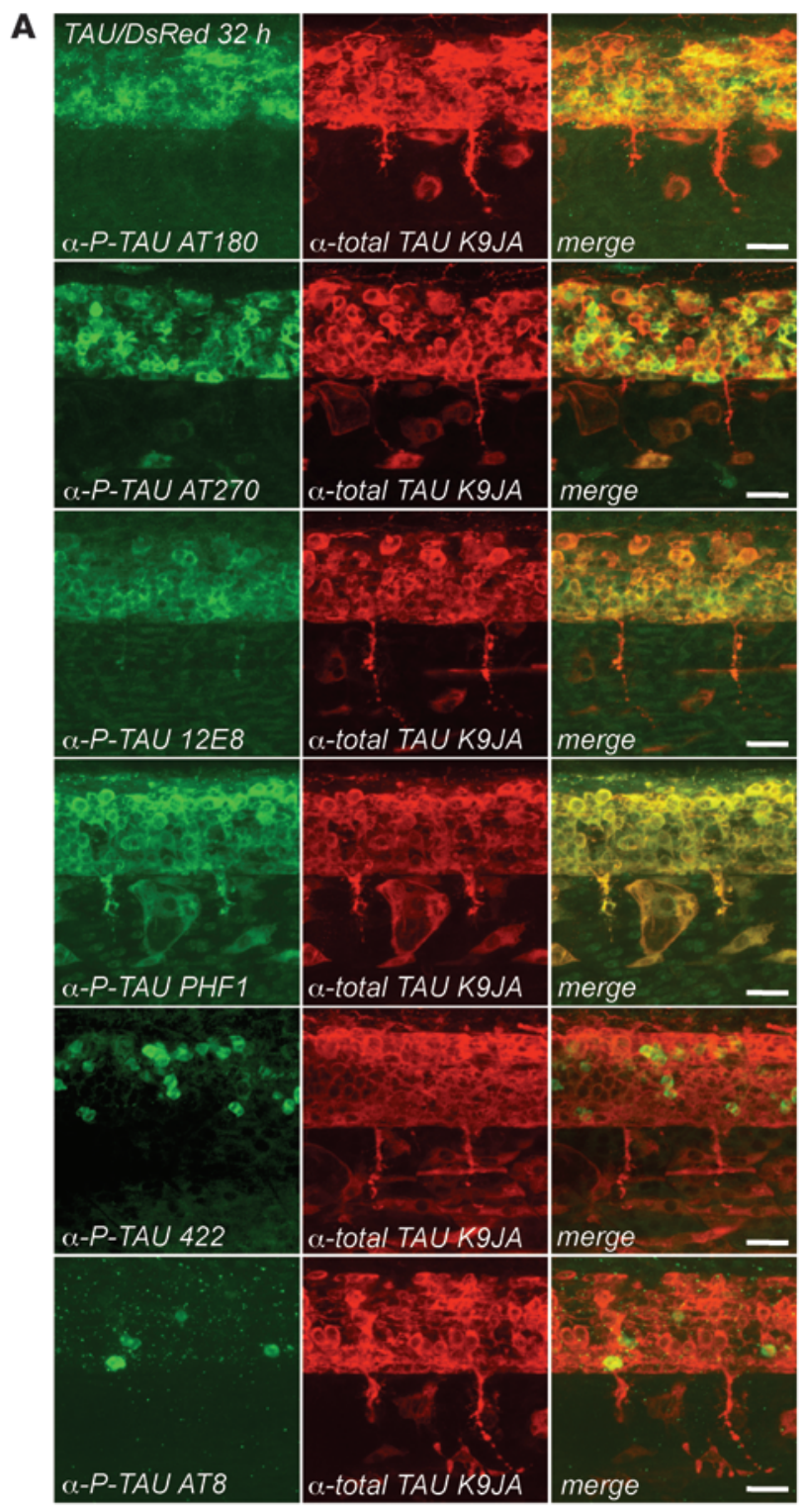

B
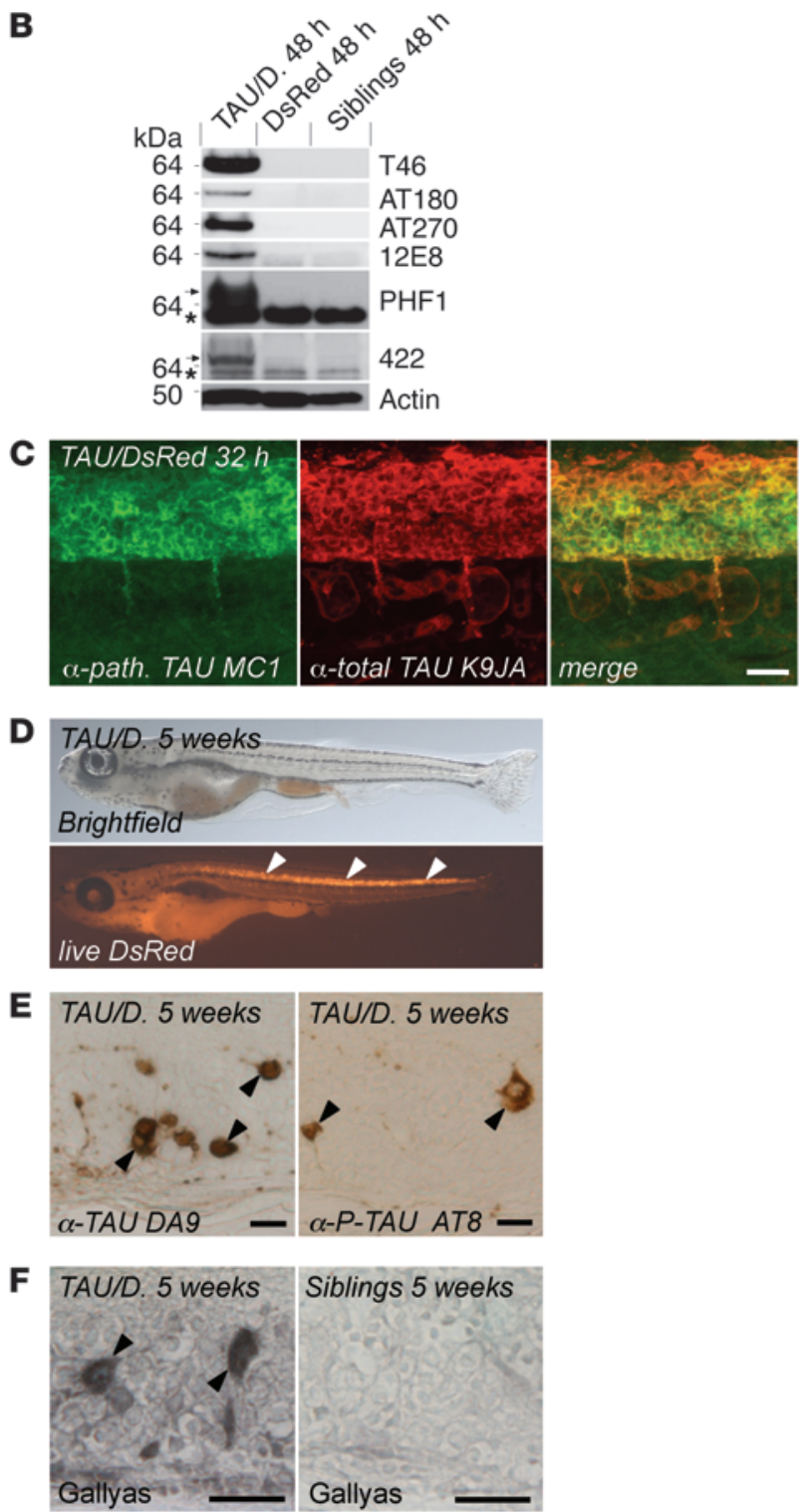

Figure 2

Expression of hTAU-P301L induces rapid pathological hyperphosphorylation, conformational changes, and aggregation of TAU in transgenic zebrafish. (A) Double whole-mount immunostainings for phosphorylated and total TAU of 32-hour-old transgenic zebrafish embryos expressing hTAU-P301L and DsRed. TAU is phosphorylated in spinal cord neurons at residues Thr231/Ser235 (AT180), Thr181 (AT270), Ser262/Ser356 (12E8), Ser396/Ser404 (PHF1), Ser422 (422), and Ser202/Thr205 (AT8). (B) WBs of total and phosphorylated TAU of 48-hour-old transgenic zebrafish embryos expressing hTAU-P301L and DsRed or DsRed alone or nontransgenic siblings. Phosphorylated TAU is only detected in TAU/DsRed transgenic embryos. No cross-reacting bands are detectable in controls at the same molecular weight. In addition to the specific band above $64 \mathrm{kDa}$ (arrow) in TAU-positive embryos, antibodies PHF1 and 422 detect a nonspecific band at lower molecular weight in all embryos (asterisk). (C) TAU changes its conformation to a pathologic state, as shown in whole-mount immunostainings, by using the conformation-specific antibody MC1, in most neurons of 32-hour-old embryos expressing the TAU transgene. (D) Side views of 5-week-old living zebrafish in bright field and DsRed illumination. The fish are still rather translucent, and the transgene-expressing cells can be detected by their red fluorescence (arrowheads). (E) Immunohistochemical stainings of spinal cord paraffin sections of the same 5-week-old TAU transgenic zebrafish for total TAU (antibody DA9) and pathologically phosphorylated TAU (AT8) (arrowheads). (F) In addition, tangles are observed by Gallyas silver staining in sections of the same 5-week-old TAU transgenic zebrafish (arrowheads). Scale bars: $20 \mu \mathrm{m}$.

422 and AT8, in 32-hour-old transgenic zebrafish. While the epitopes that are detected by early marker antibodies are present in most TAUexpressing cells, the signals of the late markers can only be found in a small subset of neuronal cells (Figure 2A). This observation raises the question of whether there is a progression to advanced pathology that these immunopositive neurons have already reached in contrast to the surrounding neurons and whether other neurons will follow later. To monitor progression of the pathological AT8 immunoreactivity, we compared the number of positive neurons in 24-hour-old, 32-hour-old, 48-hour-old, and 7-day-old embryos from a single trans- 
A

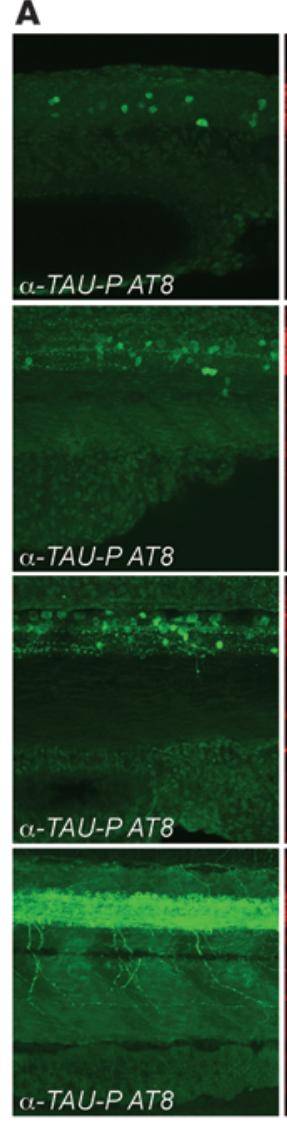

Late

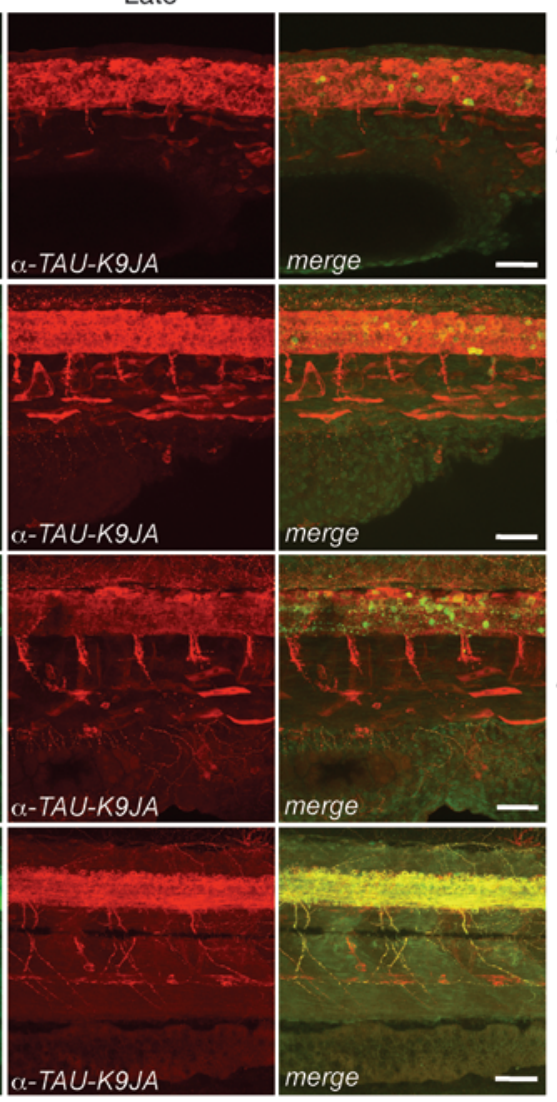

B

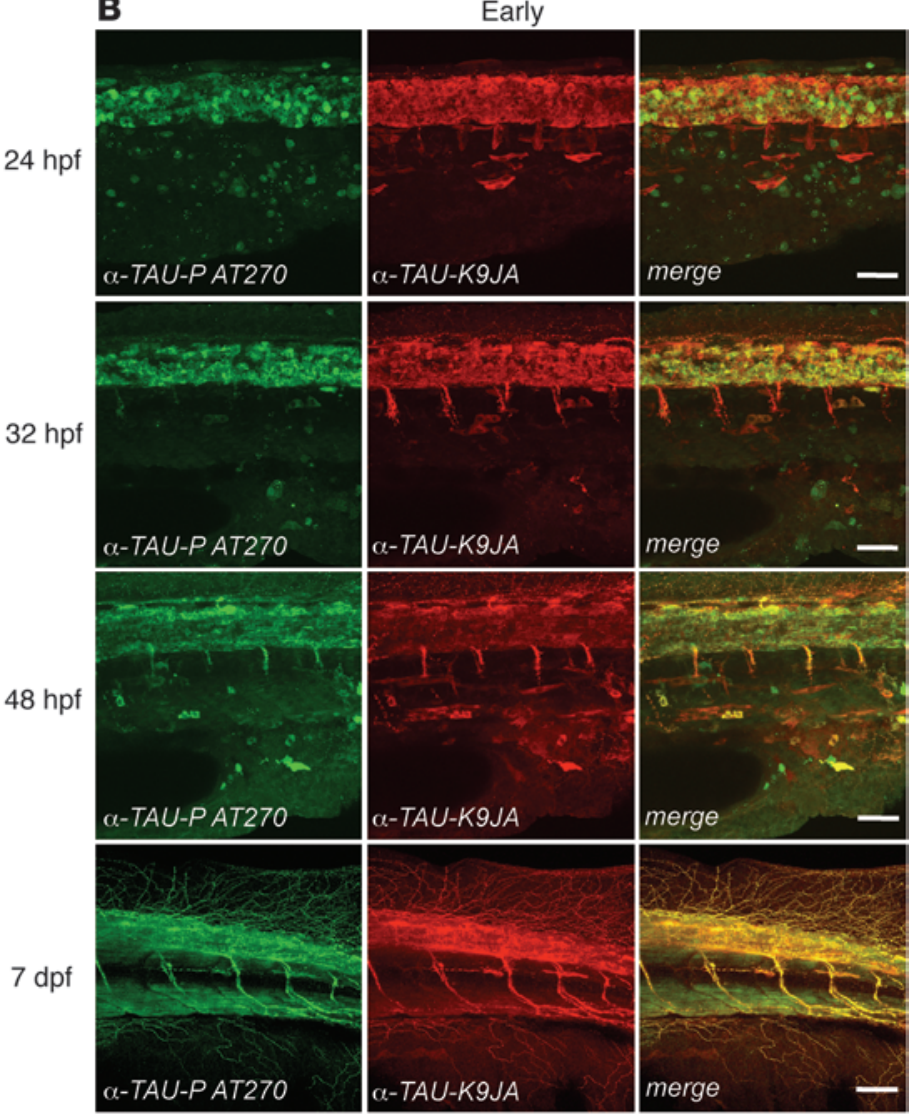

Figure 3

Rapid progression of AT8-positive late-stage FTD/AD-like pathology in transgenic embryos. (A) Double whole-mount immunostainings for TAU phosphorylated at the AT8 epitope (a late marker of pathology) and the AT270 epitope (an early marker) together with staining by antibody K9JA, which shows expression of total TAU in 24-, 32-, and 48-hpf and 7-day-old transgenic zebrafish embryos. There are very few AT8-positive neurons in the spinal cord of 24-hour-old embryos, while this number increased significantly in 32-hour-old embryos and rose even further in 48-hour-old embryos. Finally, all TAU-expressing neurons contained the AT8 epitope in 7-day-old larvae. (B) In contrast, immunoreactivity of the early marker AT270 was already strong in many TAU-expressing cells at $24 \mathrm{hpf}$ and became only slightly stronger at older stages. The level of total TAU detected in the same embryos by double staining with the K9JA antibody was similar between $24 \mathrm{hpf}$ and $32 \mathrm{hpf}$. After $48 \mathrm{hours,} \mathrm{a}$ substantial part of TAU was transferred to the neuronal projections. In contrast, AT8-positive TAU remained mainly in the cell bodies. The AT8positive somata combined with a lack of strongly AT8-positive neuronal projections is consistent with the pathological accumulation of modified TAU in neuronal cell bodies of patients. Lateral views of the trunk above the end of the yolk extension, anterior to the left. Scale bars: $50 \mu \mathrm{m}$.

genic line, with comparable levels of transgene expression. We could indeed observe a rapid and robust increase in AT8-positive neurons over this short period of time (Figure 3A). This was not the case for AT270-positive cells (Figure 3B).

Increased cell death in hTAU-expressing zebrafish neurons. Neuronal cell death is the ultimate reason for the neurological deficits of AD and FTD patients. We have therefore engaged in analysis to determine whether neurons indeed degenerate when TAU is expressed in transgenic zebrafish. Cell death can be monitored in living fish by incubation in acridine orange (27), a dye that stains nucleic acids in dying cells. We have compared 6-day-old fish expressing TAU/DsRed to DsRed only-expressing fish and nonexpressing siblings. Strikingly, we could detect a significant increase of cell death in the whole spinal cord of TAU-expressing fish, as compared with both controls (Figure 4, A-D).

To analyze neurodegeneration in more detail and to demonstrate the suitability of zebrafish larvae to study cellular processes in a whole living animal in vivo, we monitored the neurodegeneration in TAU transgenic fish by confocal time-lapse imaging. We recorded neurons in the spinal cord that express DsRed over a period of 12 hours (typical examples of still images are shown in Figure 4E; see also Supplemental Video 1) and searched for dying cells by monitoring uptake of acridine orange. An intact neuron in the field of view first altered its shape and started to round up (54.0 to 58.1 hours post fertilization [hpf]). Subsequently, this cell fragmented and took up acridine orange, indicating a breakdown of the cellular membranes (58.1 to $60.3 \mathrm{hpf}$ ), and eventually began to disappear (60.3 to $65.7 \mathrm{hpf}$ ). To our knowledge, this is the first demonstration of in vivo cell death imaging in the field of neurodegeneration.

Expression of $h T A U-P 301 L$ causes neuronal and behavioral abnormalities. It has been previously demonstrated that elevation of TAU leads to inhibition of intracellular transport with toxic consequences, which are particularly pronounced in long-projecting motoneurons $(28,29)$. This is accompanied by elevated pathologic phosphorylation. To investigate abnormalities in neuronal morphology, we stained TAU transgenic fish and control fish with antibody znp1, which labels 

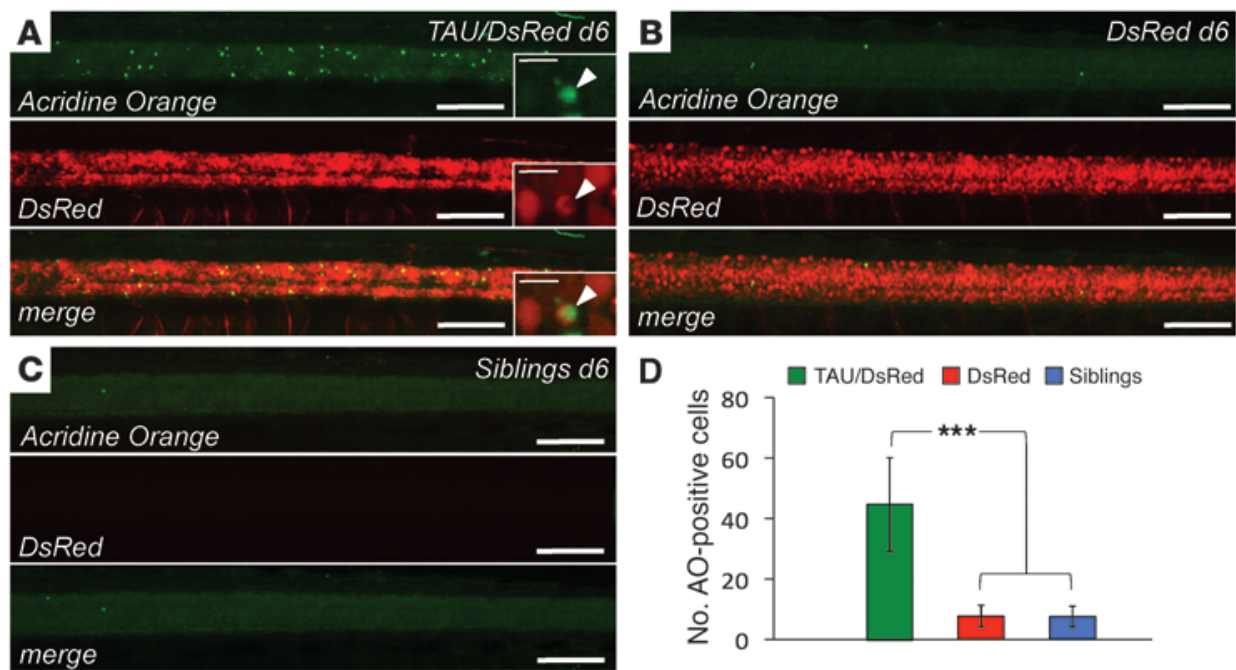

\section{D}

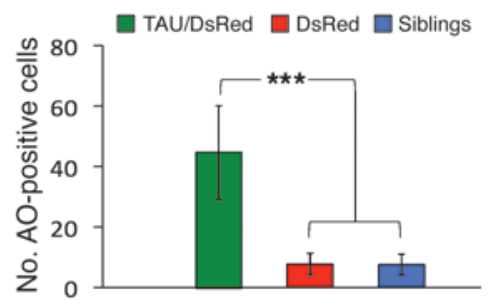

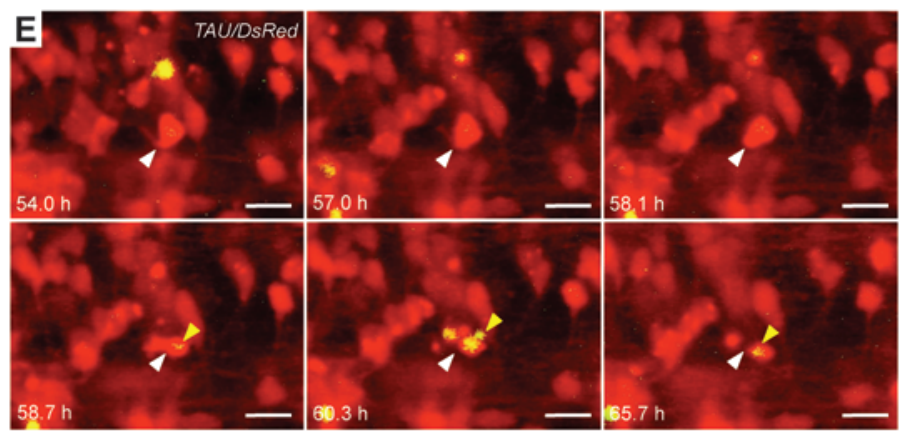

\section{Figure 4}

In vivo imaging of neuronal cell death in TAU-expressing zebrafish. (A-D) Side views of 6-day-old living zebrafish larvae expressing TAU/DsRed (A, see single acridine orange-positive neuron in inset, depicted by arrowhead), only DsRed (B), or no transgene (siblings, C) stained with acridine orange. TAUexpressing fish show substantial cell death while DsRed-expressing and nontransgenic fish show only a low, basal amount of dying cells (quantified in D). AO, acridine orange. Data represent mean \pm SD. ${ }^{\star \star \star} P<0.01$. Scale bars: $100 \mu \mathrm{m} ; 10 \mu \mathrm{m}$ (insets). (E) Still images of several time points of a time-lapse video, showing a close-up of TAU-expressing neurons in the spinal cord of transgenic zebrafish, which were stained with acridine orange. An intact neuron (white arrowhead) with an axon first changes its shape and rounds up. Subsequently, fragmentation and uptake of acridine orange is observed (yellow arrowhead; see also Supplemental Video 1). Scale bars: $10 \mu \mathrm{m}$. the synaptic protein synaptotagmin in extending axons of all primary motoneurons $(30,31)$. We measured the length of the first 4 outgrowing caudal primary $(\mathrm{CaP})$ motoneurons anterior to the end of the yolk extension, which leave the spinal cord ventrally and grow around the muscle in a stereotypic, time-dependent manner as judged by synaptotagmin (znp1) staining (32). The length of the developing motoneurons, in which synaptotagmin is present, is substantially shorter at $28 \mathrm{hpf}$ in TAU transgenic fish (Figure 5, A-C). In a later stage at $48 \mathrm{hpf}$, distribution of synaptotagmin is still altered (Figure 5, D and E), although the projections have grown further. Seven-day-old larvae are able to swim and catch food (data not shown). On the cellular level, the motoneurons in both control and TAU fish have grown around the muscle to the midline (Figure 5, F and G); however, the fine projections to the muscle are still reduced in the TAU transgenic fish in comparison with controls.

Consistent with the altered motoneuron morphology, we observed behavioral deficits, such as slow or absent movement in most of the larvae at $48 \mathrm{hpf}$, an age at which a stereotypic escape response behavior can be evoked by applying a touch stimulus to the animals. We have collected pools of transgenic fish expressing high levels of TAU/DsRed and quantified the escape response of 50 randomly picked individual larvae at $48 \mathrm{hpf}$. While most transgenic DsRed only-expressing fish with comparable expression levels or nontransgenic fish responded to a touch stimulus at the dorsal tip of the tail with a stereotypic escape response, most TAU-expressing fish show a significantly reduced or even absent response (Figure 5, H, I, and J; see also Supplemental Video 2). We hypothesize that the motoneuron defect is the reason for the observed movement deficits at this age.
Generation of potent GSK3 3 inhibitors by structure-based design. Our transgenic zebrafish rapidly recapitulated key disease markers for tauopathies, including hyperphosphorylation. We therefore determined whether our fish would be a useful tool for developing compounds that could delay or stop the pathology of tauopathies. To identify inhibitors of TAU kinases by structure-based design, we refined a previously described approach (14) and found approximately 2000 actives in a high-throughput screening campaign against the human GSK3 $\beta$ enzyme. The actives were confirmed for dose response and encompassed hits from multiple chemical series. Several compounds from the pyrazine chemical series were cocrystallized with the GSK3 $\beta$ protein. Data from $x$-ray analysis of the binding of the inhibitors within the ATP pocket of the GSK3 $\beta$ protein led to the optimization of potency and selectivity of the pyrazines. Since CDK2 is the closest homolog to GSK3 $\beta$, we optimized for CDK2 selectivity aided by structure-based design. By optimizing against CDK2, pan-kinase selectivity (27 kinases) was also obtained. This understanding of structural activity relationships subsequently led to the design of potent and selective GSK3 $\beta$ inhibitors AR-164 and AR-534 (Figure 6, A-C). AR-164 and AR-534 inhibit recombinant human GSK3 $\beta$ with Ki values (the concentration of inhibitor that reduces the formation speed of the metabolite by $50 \%$ at low substrate concentration) of $8.9 \mathrm{nM}$ and $2 \mathrm{nM}$ respectively. In contrast, AR-164 and AR-534 do not significantly inhibit CDK2/ cyclin $\mathrm{E}$ (Kis of $1440 \mathrm{nM}$ and $100 \mathrm{nM}$, respectively), demonstrating at least a 50-fold selectivity versus CDK2. Both inhibitors were cell permeable. The permeability coefficients, which were determined in $\mathrm{Caco} 2$ cells and in an in vitro BBB assay, were $11 \times 10^{-6} \mathrm{~cm} / \mathrm{s}$ and $3.9 \times 10^{-3} \mathrm{~cm} / \mathrm{min}$, respectively, for AR-164 and $20 \times 10^{-6} \mathrm{~cm} / \mathrm{s}$ and 

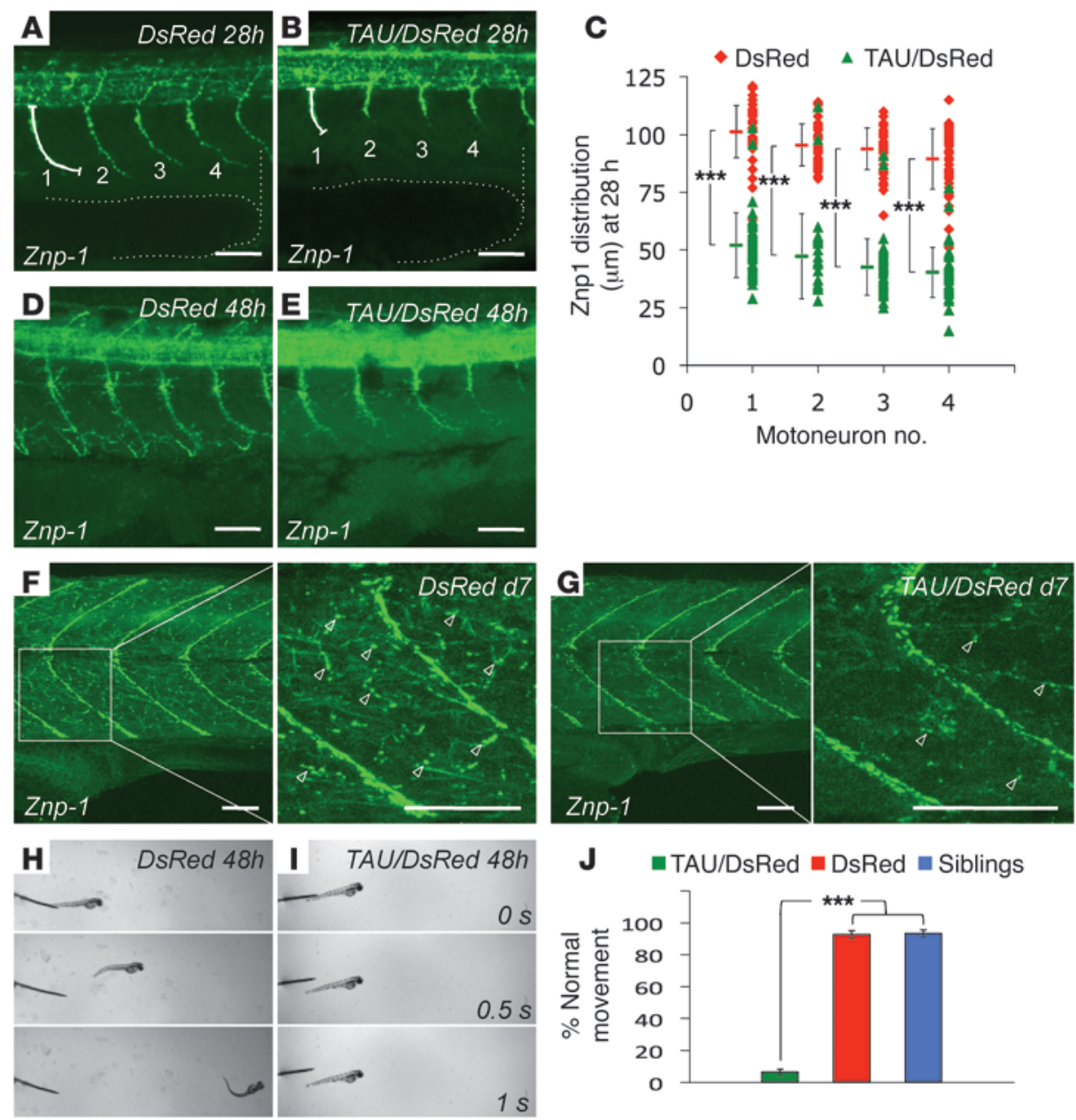

\section{Figure 5}

Expression of hTAU-P301L causes abnormalities in neuronal morphology and behavior. (A-G) Whole-mount immunostainings for znp1, which labels synaptotagmin in the extending axons of primary motoneurons. Expression of TAU causes a dramatically reduced extension of znp1positive motoneurons already at $28 \mathrm{hpf}(\mathbf{A}$ and $\mathbf{B})$. The difference in motoneuron length is quantified (C) by measuring the length of the first 4 znp1-stained motoneuron projections (marked 1, 2, 3, 4 in A and B) before the end of the yolk extension (marked by dotted line). Triangles and diamonds represent values from individual motoneurons; colored horizontal lines represent mean \pm SD. ${ }^{* \star *} P<0.01$. In 48 -hour-old embryos, the motoneurons have grown further in both TAU fish and controls, but the motoneuron extensions are still reduced (D and E). 5 days later, the motoneurons have grown around the muscle in both TAU fish and controls ( $\mathbf{F}$ and $\mathbf{G}$ ). The fine projections of motoneurons (see enlarged insets, arrowheads), are still highly reduced in TAU transgenic fish. Lateral views of the trunk above the end of the yolk extension, anterior to the left. Scale bars: $50 \mu \mathrm{m}$. $(\mathbf{H}-\mathbf{J})$ The stereotypic escape response, which is normal in DsRed-expressing $(\mathbf{H})$ and nontransgenic larvae at $48 \mathrm{hpf}($ data not shown), is highly reduced or absent in TAU-expressing larvae (I; see also Supplemental Video 2). The phenotype was quantified in groups of 50 TAU/DsRed versus DsRed transgenic larvae, which were pooled from several clutches and selected for strong and comparable DsRed expression $(\mathbf{J})$. Error bars represent mean $\pm \mathrm{SD} ;{ }^{\star \star *} P<0.01$

$12 \times 10^{-3} \mathrm{~cm} / \mathrm{min}$ respectively for AR-534 (Figure 6, B and C). These data suggest that the bioavailability of AR-534 in the brain is most likely higher than that of AR-164. Both AR-164 and AR-534 inhibit the phosphorylation of TAU at Ser396 in a dose-dependent fashion in $3 \mathrm{~T} 3$ fibroblasts engineered to stably express 4-repeat TAU protein, exhibiting $\mathrm{IC}_{50}$ values of $49 \mathrm{nM}$ and $79 \mathrm{nM}$, respectively (Figure $6, \mathrm{D}$ and $\mathrm{E})$. Effects were compared with those of the previously published GSK3 inhibitors SB-415286 (33) and lithium chloride, which exhibited $\mathrm{IC}_{50}$ values of $6 \mu \mathrm{M}$ and $1.5 \mathrm{mM}$, respectively, in our assay. Although AR-164 and AR-534 potently block phosphorylation of human TAU expressed in cell culture and display a sufficiently good $\mathrm{BBB}$ permeability in our in vitro test, this does not necessarily predict that the compounds are also active in a whole organism. Vali- dating the in vivo activity of the inhibitors in TAU transgenic mice, which were the only available vertebrate models of tauopathies so far, is an expensive and time-consuming process and not amenable to high-throughput screening for optimizing compounds for determining structural activity relationships. We have therefore studied the conservation of the targeted enzyme in zebrafish, in which drug screening would be more feasible, and found that GSK3 $\beta$ is highly conserved, with over $90 \%$ identity at the amino acid level (Figure $6 \mathrm{~F})$. Furthermore, the residues of the active site, which interact with our inhibitors, are completely identical. This high conservation to the human GSK3 $\beta$ kinase allows using the zebrafish as a model to validate the in vivo activity of GSK3 $\beta$ inhibitors and helps to further develop this lead structure. 
A
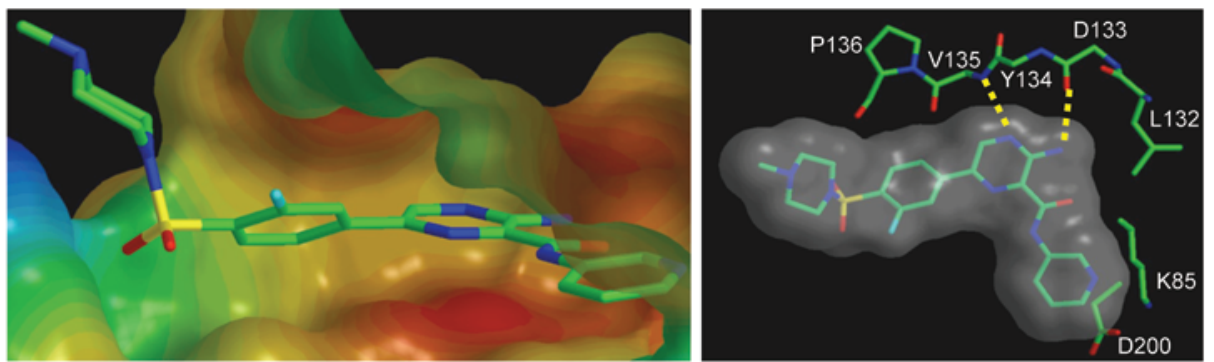

B<smiles>CN1CCN(S(=O)(=O)c2ccc(-c3cnc(N)c(C(=O)Nc4cccnc4)n3)cc2F)CC1</smiles>

AR-164: Ki GSK3: 8.9 nM

Fold selectivity over CDK2: 162

Solubility (water): $108 \mathrm{nM}$

Caco2 permeability: $11 \times 10^{-6} \mathrm{~cm} / \mathrm{s}$

BBB permeability: $3.9 \times 10^{-3} \mathrm{~cm} / \mathrm{min}$
D
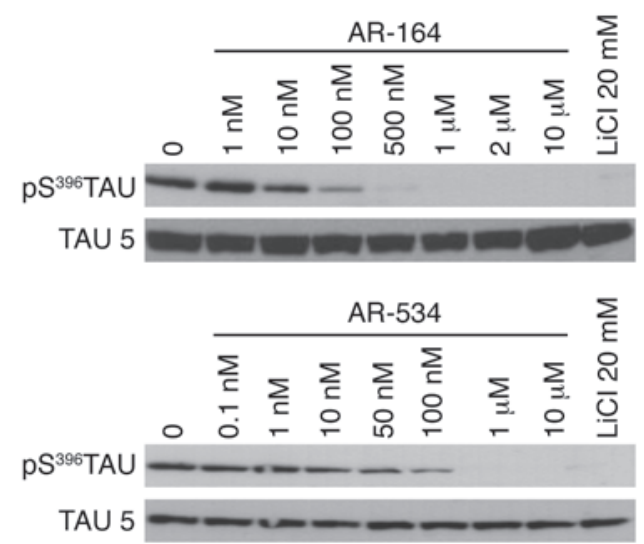

C<smiles>COCCNS(=O)(=O)c1ccc(-c2cnc(N)c(C(=O)Nc3cccnc3)n2)cc1</smiles>

AR-534: Ki GSK3: 2 nM

Fold selectivity over CDK2: 50

Solubility (water): $55 \mu \mathrm{M}$

Caco2 permeability: $20 \times 10^{-6} \mathrm{~cm} / \mathrm{s}$

BBB permeability: $12 \times 10^{-3} \mathrm{~cm} / \mathrm{min}$
E

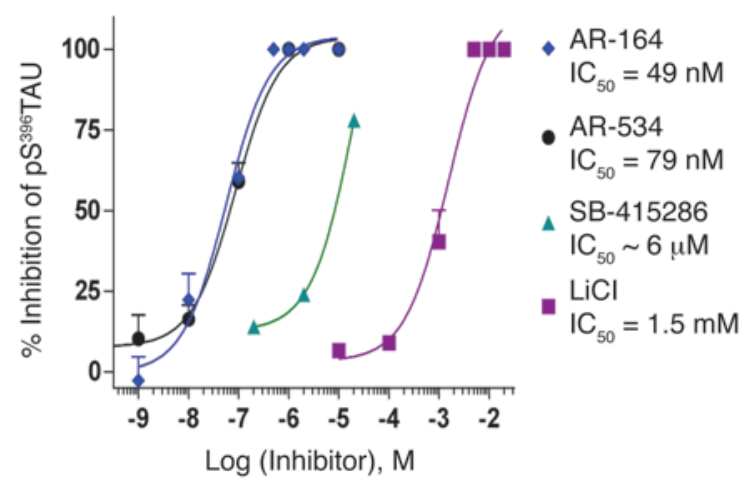

$\mathbf{F}$

Human GSK $3 \beta$ zebrafish GSK $3 \beta$

Human GSK $3 \beta$

zebrafish GSK $3 \beta$

Human GSK $3 \beta$

Zebrafish GSK $3 \beta$

Human GSK3B

zebrafish GSK $3 \beta$
MSGRPRTTSFAESCKPVQQPSAFGSMKVSRDKDGSKVTTVVATPGQGPDRPEVSYTDTKVIGNGSFGVVYQAKLCDSGELVAI KKVLQDKRFKNRELQIMRKLD 105 MSGRPRTTSFAESCKPVPQPSAFGSMKVSRDKDGSKVTTVVATPGQGPDRPQEVSYTDTKVIGNGSFGVVYQAKLCDSGELVAIKKVLQDKRFKNRELQIMRKLD 105 *

active site

HCNIVRLRYFFYSSGEKKDEVYLNLVLDYVPETVYRVARHYSRAKQTLPVI YVKLYMYQLFRSLAYIHSFGICHRDI KPQNLLLDPDTAVLKLCDFGSAKQLVRG 210 HCNIVRLRYFFYSSGDKKDEVYLNLVLDYVPETVYRVARHYSRAKQTLPMVYVKLYMYQLFRSLAYIHSFGICHRDIKPQNLLLDPDTAVLKLCDFGSAKQLVRG 210 L132-P136 D200

EPNVSYICSRYYRAPELIFGATDYTSSIDVWSAGCVLAELLLGQPIFPGDSGVDQLVEIIKVLGTPTREQIREMNPNYTEFKFPQIKAHPWTKVFRPRTPPEAIA 315 EPNVSYICSRYYRAPELIFGATDYTSSIDVWSAGCVLAELLLGQPIFPGDSGVDQLVEI I KVLGTPTREQIREMNPNYTEFKFPQIKAHPWTKVFRPRTPPEAIA 315 LCSRLLEYTPTARLTPLEACAHSFFDLRDPNVKLPNGRDTPALFNFTTQELSSNPPLATILIPPHARIQAAASTPTNATAASDANTGDRGQTNNAASASASNST- 421 LCSRLLEYTPTARLTPLEACAHSFFDELREPNVKLPNGREKPSLFNFTTQELSSNPTLASILIPAHARNQAGASTPTNPSATSDANSGDRSQTTTAASASASNTST 421 LCSRLLEYTPTARLTPLEACAHSFFDELREPNVKLPNGREKPSLFNFTTQELSSNPTLASILIPAHARNQAGASTPTNPSATSDANSGDRSQTTTAASASASNTST

\section{Figure 6}

Chemical structure, design, and characteristics of GSK3 inhibitors AR-164 and AR-534. (A) Left panel shows surface representation of the x-ray structure of GSK3 $\beta$ with inhibitor AR-164 in the active site. Polar areas are colored blue and lipophilic areas red. The methylpiperazine sulfone amide extends out toward the solvent area. Right panel shows top view of the active site of GSK3 $\beta$. Dotted yellow lines represent hydrogen bonds between the protein backbone and AR-164. The 6-membered aromatic pyrazine moiety binds together with its anilino function to the backbone of the kinase. The pyridine ring binds to the conserved salt bridge formed by Lys85 and Asp200 (x-ray resolution: $2.47 \AA$ ). AR-534 binds in a similar way (not shown). (B and C) Chemical structure of AR-164 and AR-534 and compound characterization values including Ki (mean from 3 independent experiments performed in duplicate), selectivity over CDK2, solubility and permeability coefficient $\mathrm{Pe}(\mathrm{cm} / \mathrm{min})$ over cell membranes (Caco2 cells), and BBB. (D) AR-164 and AR-534 inhibit pSer396 TAU phosphorylation in 3T3 fibroblasts harvested at 4 hours after treatment in comparison with total TAU, as analyzed by quantitative WB. (E) Graphical representation with IC 50 values of AR-164 and AR-534 effects on inhibition of TAU phosphorylation compared with SB-415286 and LiCl. (F) Alignment of protein sequences of human and zebrafish GSK3 3 . Over $90 \%$ of the amino acids are identical; the residues in the active site of the enzyme, which interact with the inhibitors, are completely conserved. 
A
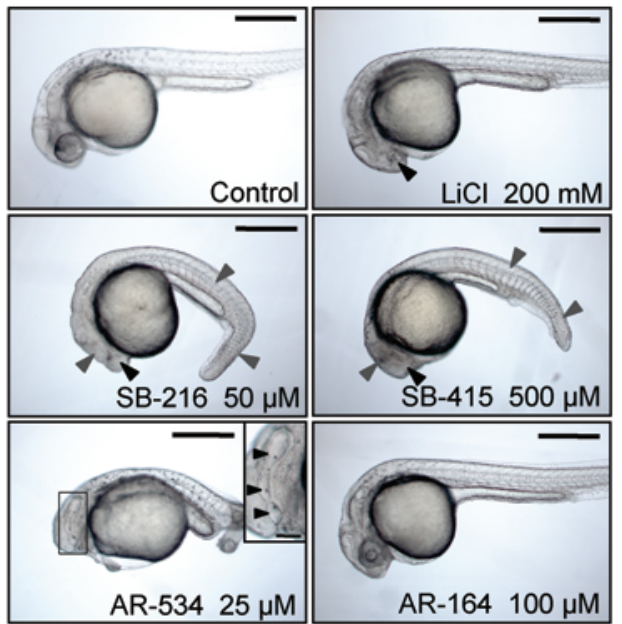
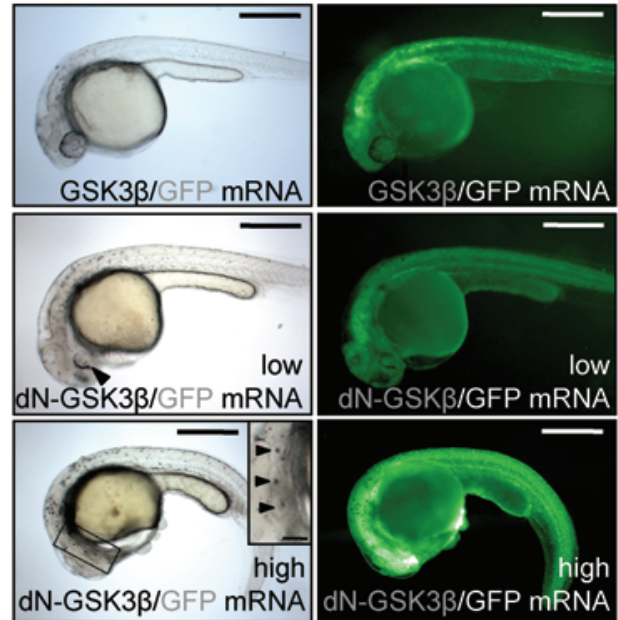
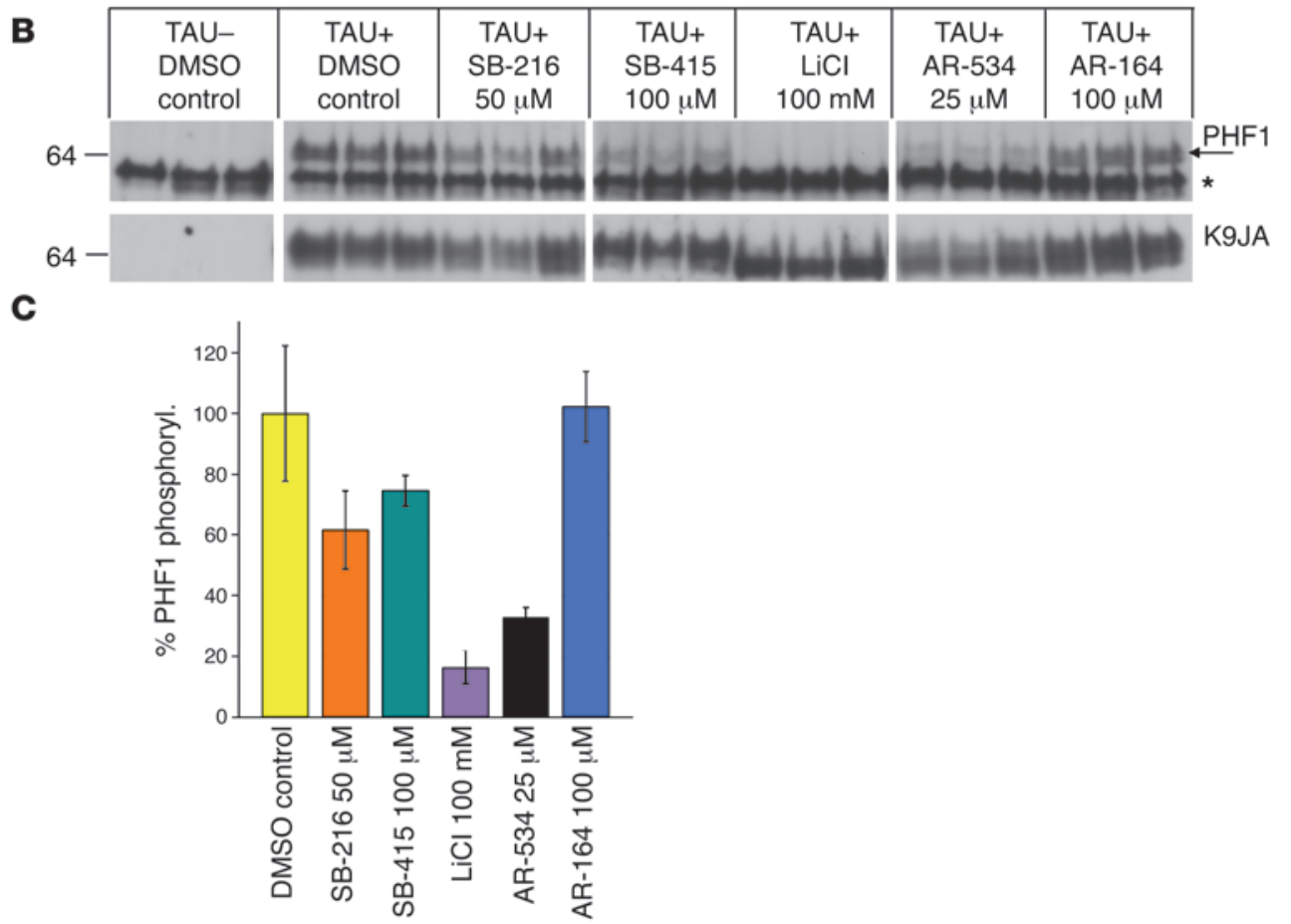

Figure 7

GSK3 inhibitors phenocopy a genetic loss of GSK3 $\beta$ and reduce pathologic hyperphosphorylation of TAU in vivo. (A) GSK3 inhibitors have specific effects on early wild-type zebrafish development when treatment occurs between 4 and $24 \mathrm{hpf}$. LiCl treatment caused strongly reduced eye formation (black arrowheads), while SB-216763 and SB-415286 also perturbed formation of brain, somites, and tail (gray arrowheads). In addition to these phenotypes, AR-534 also caused multiple ear formation (arrowheads in enlargement). In contrast, AR-164, which has in vitro activity comparable to that of AR-534, did not cause any detectable change in phenotype even at 4-fold concentration. Embryos injected with dN-GSK3 $\beta$ mRNA to suppress the function of endogenous GSK3 $\beta$ phenocopied alterations of compound-treated fish; however, only AR-534 was potent enough to phenocopy the ear multiplication seen in embryos expressing high levels of dN-GSK3 $\beta$ (arrowheads in enlargement). Embryos injected with wild-type GSK3 $\beta$ did not display a phenotype at comparable mRNA concentrations. Scale bars: $500 \mu \mathrm{m} ; 100 \mu \mathrm{m}$ (insets). (B) GSK3 inhibitors reduce pathologic TAU phosphorylation in vivo when applied to TAU transgenic fish between 20 and $100 \mathrm{hpf}$. Phosphorylated TAU was detected by WB using antibody PHF1 and normalized to total TAU (K9JA). The band intensities were compared with a DMSO control on the same gel. The upper specific PHF1-band (arrow) was quantified. As already shown in Figure 2, the lower PHF1 band was unspecific (asterisk). (C) Quantification of WB band intensities of phospho-specific TAU as a percentage of the total amount of TAU detected by K9JA shown in B.

Potent in vivo effects of a newly developed GSK3 $\beta$ inhibitor. After demonstrating that the TAU transgenic zebrafish display a number of pathological symptoms that are diagnostic for tauopathies, we used the fish to investigate in vivo 2 known GSK3 inhibitors, SB-216763 and SB-415286 (33), as well as our 2 newly developed compounds, AR-534 and AR-164, which have a strong and selective inhibitory activity on GSK3 $\beta$ in vitro (see Figure 6). In addition, we treated transgenic fish with $\mathrm{LiCl}$ as described (34), as a positive control. 
To evaluate whether the compounds affect zebrafish Gsk3 $\beta$ activity, we first treated wild-type zebrafish embryos between 4 and $24 \mathrm{hpf}$ and examined their developmental phenotype. GSK3 $\beta$ is a component of the canonical Wnt pathway that is required for many early patterning events in the developing vertebrate embryo. Disturbances of this pathway therefore lead to characteristic developmental defects (35). LiCl treatment caused strongly reduced eye formation, as previously described (36), while SB-216763 and SB-415286 also perturbed the normal formation of brain, somites, and tail in addition to the eye phenotype (Figure 7A). The inhibitor AR-534 had an even more drastic effect on the fish, causing multiple ear formation in addition to the described phenotypes. In contrast, AR-164, which has a comparable in vitro activity to AR-534, did not cause any detectable phenotype even at 4-fold higher concentration (Figure 7A). To compare the efficiency of the inhibitors and the specificity of the evoked phenotypes with a genetic loss of the endogenous enzyme function, we injected mRNA encoding dominant-negative Xenopus GSK3 $\beta$-K85R (dN-GSK3 $\beta$ ), which is over $92 \%$ identical to zebrafish Gsk3 $\beta$, into zebrafish eggs. To monitor dosedependent effects, we coinjected dN-GSK3 $\beta$ mRNA and GFP mRNA and used GFP fluorescence to select embryos with lower or higher protein expression. Strikingly, embryos expressing lower amounts of $\mathrm{dN}$-GSK3 $\beta$ phenocopied the eye loss, which was also observed in embryos treated with SB-216763 and SB-415286 and LiCl, whereas only the embryos expressing higher amounts also phenocopied the multiple ear formation monitored in AR-534-treated embryos (Figure 7A). This result indicates that AR-534 has the highest specific in vivo efficacy of all compounds used in this study. Similar to $\gamma$-secretase inhibitors, which cause very profound defects in Notch signaling during early development of zebrafish (37), such inhibitors may only be used during late adulthood when FTD and AD develop, thus excluding their negative effects on embryonic development. To confirm that treatment of larvae at later stages does not cause deleterious developmental problems but reduces pathologic TAU phosphorylation in vivo, we now treated TAU transgenic fish for 3 days starting at $20 \mathrm{hpf}$, after early development was completed. Treatment starting at this time point did not cause any major morphological phenotype to the larvae (data not shown). After the treatment period, embryos were lysed and subjected to quantitative Western blot (WB) analysis, in which the levels of phosphorylated TAU were normalized to total TAU to exclude differences in expression levels or gel loading. SB-216763 and SB-415286 reduced phosphorylation of TAU at the disease-specific PHF1 epitope by $30 \%$ to $40 \%$, a low activity in comparison with the nonspecific inhibitor $\mathrm{LiCl}$, which reduces phosphorylation by nearly $90 \%$ when used at high concentration (Figure 7, B and C). AR-534 was more potent than the existing specific inhibitors SB-216763 and SB-415286 and reduced TAU phosphorylation by $70 \%$ (Figure 7, B and C). In contrast to the other inhibitors, AR-164 did not show any inhibitory activity on TAU phosphorylation in vivo (Figure 7B), which is consistent with both its lack of activity on early development and the lower penetrability in the in vitro BBB screen, as shown in Figure 6. We conclude that, consistent with its activity on early zebrafish development, AR-534 is also the most potent specific inhibitor of GSK3 $\beta$-mediated TAU phosphorylation.

\section{Discussion}

We provide a newly developed technology approach for the fast and efficient generation of transgenic zebrafish to study human diseases resulting from protein misfolding and/or overexpression and to optimize promising drug candidates in vivo. The generation of transgenic zebrafish overexpressing high levels of human proteins has so far been rather difficult. We have overcome these limitations with the efficient Tol2 transposon and Gal4/UASbased expression of human TAU-P301L in transgenic zebrafish. In contrast to previous studies $(11,12)$, we could monitor early pathology, including disease-specific hyperphosphorylation and conformational changes of TAU as well as neuronal and behavioral abnormalities within the first 2 days of embryonic development in stable transgenic zebrafish. Furthermore, the larvae developed substantial neurodegeneration after a few days. These phenotypes appear much more rapidly in our fish model than in the existing mouse models, although zebrafish kept under laboratory conditions live as long as 3 to 5 years (38), which is comparable to laboratory mice (39). Importantly, this early pathology is crucial to fully exploit all advantages of the zebrafish system, such as optical clarity and ease of manipulation. The similarity of the evoked phenotypes to human disease is completed by the appearance of neurofibrillary tangles after 5 weeks, validating our fish as a wellsuited model for tauopathies in vivo.

We expect the transgenic zebrafish to become a valuable model system for gaining further insights into the pathology of dementias by exploiting the unmatched potential for the analysis of cellular processes in the living organism, which the zebrafish offers in contrast with other vertebrate models. Although living cells can also be monitored in transgenic mice, experiments in the zebrafish can be performed in a noninvasive way in much higher spatial and temporal resolution. Whole-cell imaging of neurons comprising somata and projections can be done in embryos and larvae over several days without removing the specimen from the microscope (40). Since FTD-like pathology can be detected already after 1 to 2 days for early and late disease markers and this pathology progresses rapidly, we can monitor disease progression in a compressed timescale in young zebrafish embryos and larvae, which are perfectly suited for in vivo imaging of neurons due to their optical transparency. In fact, confocal imaging even allowed us to follow neuronal cell death in vivo. To our knowledge, this is the first demonstration of in vivo cell death imaging in the field of neurodegeneration. Moreover, neurons can be imaged in their natural environment together with neighboring cell types such as astroglia, oligodendrocytes, and microglia, which have been shown to participate in the pathology of several neurodegenerative diseases (1). These important environmental influences are not faithfully recapitulated in tissue explants or primary cell culture. The early phenotypes allow testing for possible genetic modifiers of pathology in vivo through transient overexpression of wild-type or dominant-negative forms of proteins by DNA or RNA injection or downregulation mediated by injection of antisense oligonucleotides.

The rapid appearance of pathologic phenotypes allows use of the transgenic zebrafish larvae not only to image and understand disease processes in a living animal but also to validate and even screen on a relatively large scale for compounds that modify the pathology in vivo. The hyperphosphorylation of TAU, which our fish model develops within 32 hours after fertilization, is believed to be a key initiator of detachment of normal TAU from microtubules and subsequent oligomerization and aggregation. Preventing this phosphorylation by blocking the involved kinases could potentially slow further progression of pathology and thus be an efficient treatment option for $\mathrm{AD}$ and FTD (13). We have developed compounds that bind to the active site of GSK $3 \beta$ and effectively block enzyme activity. The identifica- 
tion of potent cell-permeable kinase inhibitors with in vivo efficacy is a key component of the drug discovery process. With the use of rational drug design, optimization of the pyrazine chemical series led to the identification of several GSK3 inhibitors. We demonstrated by in vitro studies that 2 of these compounds have high selectivity for the target enzyme, good bioavailability, and high potency to block TAU phosphorylation in cell culture. However, it is frequently observed that such inhibitors may not be active in vivo, since they, for example, do not enter the cells, are transported out, or are rapidly metabolized when tested in whole organisms (13). This emphasizes the necessity of rapid, high volume in vivo drug testing in disease models, which is not feasible in transgenic mice on a large scale. Transgenic zebrafish offer advantages of both in vitro and in vivo systems and allow rapid screening for pathology-modifying drugs on a large scale, since embryos can be tested for rescue of the pathologic phenotypes after compound treatment in a 96-well-plate scale (9).

We show that GSK3 $\beta$ inhibitors cause specific phenotypes when applied during early embryonic development and that the evoked phenotypes already indicate the in vivo potency of the inhibitor. Moreover, the GSK3 $\beta$ inhibitors can effectively reduce the rapid disease-specific hyperphosphorylation of TAU in our transgenic zebrafish. Using our model, we have evaluated the activity of 2 newly developed GSK3 $\beta$ inhibitors with comparable in vitro activity. One of the inhibitors, AR-534, was also very active in vivo, with a much higher effect than the previously described GSK3-specific inhibitors SB-216763 and SB-415286, which we used for comparison. In contrast, the inhibitor AR-164, which is equally potent in cell culture, was inactive in vivo. These data were consistent with the over 3-fold lower performance of AR-164 in our BBB test. As this is an in vitro test, which can only serve as a model of the in vivo situation, the bioavailability of AR-164 could be even lower in our transgenic zebrafish, explaining the lack of activity. It has been shown recently that zebrafish form a functional BBB that is similar to that of higher vertebrates. The BBB in zebrafish consists of endothelial cells, which can be characterized by the expression of the marker proteins claudin-5 and ZO1 and starts to become functional at 3 days after fertilization (10). The developing BBB might be less permeable to AR-164 versus AR-534 and could therefore be one reason for the absent in vivo activity of AR-164. Alternatively, degradation or metabolism of AR-164 could also account for the activity differences we observed. Taken together, the different activities of AR-534 and AR-164 in the in vitro versus the in vivo situation clearly illustrate the need for effective in vivo-screening tools, as this can help to identify promising compounds more quickly and directly, while at the same time allowing one to eliminate substances without reasonable in vivo activity early in the screening process. Taken together, our transgenic zebrafish model could be a promising tool for streamlining pharmacological screening for many neurodegenerative diseases. In fact, we have already successfully used this technology to generate transgenic zebrafish lines overexpressing a number of other amyloidogenic proteins, such as amyloid $\beta$ peptide, TDP-43, and $\alpha$-synuclein (data not shown).

\section{Methods}

\section{Animal husbandry}

All experiments were performed in accordance with animal protection standards and were approved by the government of Upper Bavaria (Regierung von Oberbayern, Munich, Germany). The zebrafish wild-type AB line was maintained, mated, and raised as described (41). Embryos were kept in E3 medium at $28.5^{\circ} \mathrm{C}$ and staged as described (42).

\section{Constructs}

Our transgenesis constructs are based on the PT2KXIGdeltaIN plasmid (a gift from K. Kawakami, National Institute of Genetics, Shizuoka, Japan), which is derived from the medaka Tol2 transposable element (16). pT2KXIGdeltaIN was cleaved by BglII and XhoI to remove the insert between the Tol2 sites, and a multiple cloning site (MCS) was introduced. The MCS was cut out by PvuII from pBS_I-SceI, which contains the pBluescript MCS flanked on both sides by I-SceI restriction sites. To assemble the final transgenesis constructs, fragments were first combined in pBS_I-SceI, as this vector contained more suitable restriction sites, and later transferred to PT2KXIGdeltaIN.

Driver construct. The SV40 late polyA signal was PCR amplified from $\mathrm{pCS}^{+}$using the primers $\mathrm{pCS} 2^{+}$poly(A)-F SacII $\left(5^{\prime}\right.$-AAAAAACCGCGGAGTCGTATTACGTAGATCCAGACATGA- $3^{\prime}$ ) and pCS2 ${ }^{+}$poly(A)-R SacI (5'-AAAGAGCTCCACACCTCCCCCTGAAC-3'), cleaved by SacI/SacII, and ligated into pBS_I-SceI. The neuronal HuC promoter (19) was PCR amplified using the primers HuC-F XhoI/EcoRI (5'-GCTCGAGGAATTCACTAATTTGAATTTAAATGC-3') and HuC-R2 EcoRI/ClaI (5'-GGAATTCATCGATTCTTGACGTACAAAGATGATATTGATCTAGG-3'), cut by XhoI/ClaI, and ligated into pBS_I-SceI_pA. Gal4-VP16 (17) was cleaved by BamHI/ SnaBI from pBS_HuC_Gal4-VP16, blunted, and ligated into pBS_I-SceI_ HuC_pA cut by EcoRV/SnaBI. The HuC-Gal4-VP16-pA fragment was transferred to pT2KXIGdeltaIN by I-SceI digest and ligation.

Responder constructs. We generated transgenic control fish expressing DsRed but not TAU using a construct that contained E1b-UAS-E1b flanked by DsRed and TAU, but TAU was isolated from the minimal E1b promoter by an insert of $200 \mathrm{bp}$ (Ins), which inhibits expression of TAU. DsRed.T4 (43) was cleaved from pCS2 ${ }^{+}$DDsRed.T4 by NcoI/SnaBI digest to replace GFP in the vector pBS_E1b_UAS_E1b_GFP_pA. This vector contains UAS flanked by E1b minimal promoters (17) on both sides. Ins-hTAU-P301L was cleaved by BglII/BamHI from pNG2htau40/P301L and ligated at the BamHI site of pBS_I-SceI_pA. E1b-UAS-E1b-DsRed-pA was cleaved by HindIII/SmaI from pBS_E1b_UAS_E1b_DsRed.T4_pA, blunted and ligated into EcoRVcleaved pBS_I-SceI_Ins_hTAU-P301L_PA. The full expression cassette was transferred to PT2KXIGdeltaIN by I-SceI digest and subsequent ligation. The final Responder construct was used together with the Driver construct to generate transgenic fish $\mathrm{Tg}(\mathrm{HuC}: \mathrm{Gal} 4 / \mathrm{UAS}-\mathrm{DsRed})$.

To generate transgenic fish expressing DsRed and TAU, hTAU-P301L was amplified from pNG2htau40/P301L by PCR using primers hTAU ATG-F BamHI/NcoI (5'-GGGATCCCATGGCTGAGCCCCGCCAGGA-3') and hTAU TAG-R NcoI/EcoRI (5'-GGAATTCCATGGTCACAAACCCTGCTTGGCTA-3'), cleaved by NcoI and inserted into pBS_E1b_UAS_ E1b_GFP_pA cleaved by NcoI/SnaBI to remove GFP. DsRed together with the SV40 late polyA signal were cleaved by ClaI/ApaI from $\mathrm{PCS} 2^{+}$ DsRed.T4 and introduced into pBS_E1b_UAS_E1b_hTAU-P301L_pA. The insert in pT2KXIGdelta-hTAU-P301L-Ins-E1b-UAS-E1b-DsRed was removed by ApaI/NheI digest and replaced by the new cassette cleaved from pBS_pA_DsRed_E1b_UAS_E1b_hTAU40-P301L_pA. The distance of TAU from E1b is only $35 \mathrm{bp}$ in this construct; therefore, TAU is efficiently expressed together with DsRed. The final Responder construct was used together with the Driver construct to generate transgenic fish $\mathrm{Tg}$ (HuC:Gal4/TAU-P301L-UAS-DsRed).

Gateway constructs. Gateway recombinations were performed according to the manufacturer's instructions (Invitrogen). Details about Gateway cloning can be found at www.invitrogen.com/site/us/en/home/Products-andServices/Applications/Cloning/Gateway-Cloning.html.

To adapt our responder construct to Gateway cloning, hTAU-P301L was replaced by a Gateway destination site flanked by attR1/2, which was cleaved from pBS-DEST_attR1-2 (a gift from P. Lemaire, IBDML, Marseille, France) by EcoRV. The resulting construct was termed Destination Responder vector. 
To adapt our Driver construct to Gateway cloning, the HuC promoter was also replaced by a Gateway destination site flanked by attR $1 / 2$. The resulting construct was termed Destination Driver vector. To test the functionality of the vectors, the $\mathrm{HuC}$ and $\alpha \mathrm{Tub}$ promoter and hTAU-P301L were amplified by PCR using the following primers: HuC-F XhoI/EcoRI (5'-GCTCGAGGAATTCACTAATTTGAATTTAAATGC-3') and HuC-R2 EcoRI/ClaI (5'-GGAATTCATCGATTCTTGACGTACAAAGATGATATTGATCTAGG-3') for HuC; M13-FP (5'-TGTAAAACGACGGCCAGT-3') and aTub-R (5'-GGCACGCTGTGAAGAAAAAG-3') for $\alpha$ Tub; and hTAU ATG-F BamHI/NcoI (5'-GGGATCCCATGGCTGAGCCCCGCCAGGA-3') and hTAU TAG-R NcoI/EcoRI (5'-GGAATTCCATGGTCACAAACCCTGCTTGGCTA-3') for hTAU-P301L. PCR products were TOPO-cloned into entry vectors using the $\mathrm{pCR} 8 / \mathrm{TOPO} / \mathrm{GW}$ Kit from Invitrogen and sequenced. Promoters and TAU were then LR recombined into Destination Driver and Responder vectors, using LR Clonase II from Invitrogen according to the manufacturer's instructions.

\section{Plasmid preparation, mRNA synthesis, and embryo injection}

Plasmids were prepared by extraction with a MACHEREY-NAGEL Maxi Kit and further cleaned up by using the Qbiogene GENECLEAN Kit according to the manufacturer's instructions. The vectors PCS-TP (a gift from K. Kawakami, National Institute of Genetics, Shizuoka, Japan), pCS2 ${ }^{+}$GFP, pCS2 ${ }^{+}{ }_{2} \mathrm{dN}-\mathrm{GSK} 3 \beta$, and pCS2 ${ }^{+}$GSSK3 $\beta$ (both gifts from R. Rupp, LudwigMaximilians-University) were linearized by NotI and cleaned up by phenol/ chloroform precipitation. mRNAs were synthesized from the linearized vectors with the Ambion mMESSAGE mMACHINE kit (Applied Biosystems) according to the manufacturer's instructions and stored in small aliquots at $-80^{\circ} \mathrm{C}$. Shortly before injection, circular Driver and Responder plasmids were mixed with Tol2 transposase mRNA, all at a final concentration of $25 \mathrm{ng} / \mu \mathrm{l}$, in $\mathrm{dH}_{2} \mathrm{O}$ containing diethylenepyrocarbonate-treated (DEPCtreated) $0.2 \mathrm{M} \mathrm{KCl}$ and $20 \%$ phenol red (44). GFP mRNA was used at a concentration of $100 \mathrm{ng} / \mu \mathrm{l}$ and GSK3 $\beta$ mRNAs were used at a concentration of $50 \mathrm{ng} / \mu \mathrm{l}$, all diluted in DEPC-treated $\mathrm{dH}_{2} \mathrm{O}$ containing $20 \%$ phenol red.

One-cell-stage embryos were collected, oriented on agar plates, and injected with approximately $1 \mathrm{nl}$ of the injection solution. Plasmid DNA and transposase mRNA were injected through the yolk into the cytoplasm of the first cell before the first cell division. GFP and GSK3 $\beta$ mRNAs were mixed before use and injected into the yolk. At around 6 to $8 \mathrm{hpf}, 2$ random fertilized embryos from each dish were tested for transposition by PCR as described (44). At $30 \mathrm{hpf}$, embryos were selected for DsRed expression under a Leica fluorescent stereomicroscope and raised at $28.5^{\circ} \mathrm{C}$ to adulthood. Adult founder fish were identified by outcrossing them to $\mathrm{AB}$ wildtype fish and screening the F1 generation for DsRed-expressing embryos. These embryos were raised to establish transgenic lines.

\section{Identification of transgenes by PCR}

The offspring of 225 embryos of an F2 outcross were analyzed by PCR. Three independent PCRs were performed to show Gal4 on the Driver construct, TAU on the responder construct, and actin as a loading control. The following primers were used: Gal4: HuC 3'F 5'-TGGCGAAGACTGTCCTTTTT-3', Gal4-R 5'-GGTCTTCTCGAGGAAAAATCAG-3'; hTAU: TAU-OF 5'-AGGAGTTCGAAGTGATGGAAGAT-3', TAU-IR 5'-GTGGCGATCTTCGTTTTACCAT-3'; and actin: actin-F 5'-TGTTTTCCCCTCCATTGTTGG-3', actin-R $5^{\prime}$-TTCTCCTTGATGTCACGGAC-3'. For the PCRs, the 4- to 24-hour-old eggs were fixed in methanol; then the DNA was extracted by digestion with proteinase $\mathrm{K}$ for 4 hours at $55^{\circ} \mathrm{C}$ followed by inactivation of the enzyme for 10 minutes at $95^{\circ} \mathrm{C}$. The extracted DNA was used as a template for PCR. To analyze the results, the products of the 3 PCRs for each possible genotype from 2 different embryos were mixed together, loaded on a gel, counted, and imaged.

\section{IF, immunobistochemical stainings, Gallyas silver stainings, and WBs}

Whole-mount IF stainings were performed according to standard methods (45). For immunohistochemical and Gallyas silver stainings on paraffin sections, the 5-week-old fish were deeply anesthetized and killed in water with ice. Then the whole fish were fixed overnight in $4 \% \mathrm{PFA}$ and transferred to PBS and the sections and stainings were performed as described for mouse tissue (46).

WBs were performed according to standard methods. Embryos or larvae were frozen in liquid nitrogen and 10 to $50 \mu \mathrm{l}$ of chilled Laemmli buffer were added per fish. Fish were homogenized by sonication and incubated at $95^{\circ} \mathrm{C}$ for 10 minutes. Embryo lysates were subjected to centrifugation at $13,000 \mathrm{~g}$ for 1 minute, and 10 to $20 \mu \mathrm{l}$ of the supernatant was loaded on an $8 \%$ or $10 \%$ polyacrylamide gel. The following antibodies were used for IF, immunohistochemistry (IHC), and WB: (a) TAU. T46 (20) (IF: 1:200, WB: 1:1000; Invitrogen), K9JA (IF: 1: 500, WB: 1:5000; no. A0024; Dako), DA9 (IHC: 1:250; a gift from P. Davies, Albert Einstein College of Medicine, New York, New York, USA), AT8 (25) (IF: 1:200, IHC: 1:50, WB 1:1000; Pierce, Thermo Scientific), 422 (24) (IF: 1:200, WB 1:1000; a gift from C. Czech, Hoffmann La Roche, Basel, Switzerland), AT180 (21) (IF: 1:200, WB: 1:1000; Pierce, Thermo Scientific), AT270 (21) (IF: 1:200, WB: 1:1000; Pierce, Thermo Scientific), 12E8 (22) (IF: 1:200, WB: 1:1000; a gift from P. Seubert, Elan Pharmaceuticals, San Francisco, California, USA), MC1 (26) (IF: 1:50; a gift from P. Davies, Albert Einstein College of Medicine, New York, New York, USA), PHF1 (23) (IF: 1:100, WB 1:1000; a gift from P. Davies, Albert Einstein College of Medicine, New York, New York, USA); (b) Other. DsRed (IF: 1:200; Clontech), actin (WB: 1:1000; Sigma-Aldrich), znp1 (30) (IF: 1:100; Developmental Studies Hybridoma Bank), Alexa Fluor 488 goat anti-mouse IgG (1:500; Invitrogen), Alexa Fluor 546 goat anti-rabbit IgG (1:500; Invitrogen), Alexa Fluor 488 goat anti-rat IgG (1:500; Invitrogen).

\section{Acridine orange stainings and time-lapse imaging}

Living zebrafish larvae were anesthetized with Tricaine (Sigma-Aldrich), incubated in a solution of $3 \mu \mathrm{g} / \mathrm{ml}$ acridine orange (Sigma-Aldrich) in E3 medium with Tricaine for 30 minutes (27), and washed twice in E3 with Tricaine. For imaging, fish were embedded in $1.6 \%$ low melting agarose in E3 and overlayed with E3/Tricaine. The green nuclei of the neurons were counted in the whole spinal cord of 44 fish for each genotype using a Zeiss compound microscope with a Zeiss Plan-Apochromat 10×/0.45 lens. For time-lapse imaging, a $z$-stack of a chosen region of the spinal cord of the transgenic fish with a thickness of $10 \mu \mathrm{m}$ was imaged every 3 minutes for 10 to 16 hours on a Zeiss LSM 510 META inverted confocal microscope (Zeiss, provided by the Hans and Ilse Breuer Foundation, Frankfurt am Main, Germany) in a heated chamber. The images of the z-stack were combined in a maximum projection, and the time series of image projections was exported to a time-lapse video with 8 frames per second using Zeiss LSM 510 Confocal software, version 4.2 SP1. The videos and still images taken from the videos were processed with Image 1.41 (http://rsb.info.nih. gov/ij/) and iMovie 7 on Mac OSX 10.5.

\section{Behavioral assays}

48-hour-old living zebrafish larvae of several clutches were pooled together and sorted for visible DsRed expression. The selected fish were dechorionated manually at least 3 hours before the experiment. To evaluate the escape response, fish were touched with the tip of a fine needle for at least 2 times at the dorsal tip of the tail. An escape response in which the fish did not move a distance of at least 3 times its own body length was considered as reduced. A minimum of 3 groups of 50 fish were quantified for each genotype. At least one experiment was recorded and processed as a video. 


\begin{abstract}
Microscopy
Living zebrafish embryos, larvae, and 5-week-old juveniles were examined and imaged on a fluorescent stereomicroscope (Leica). For imaging fixed stainings, heads of zebrafish embryos were removed, and the tails were flat mounted in $1 \%$ low melting agarose in $50 \%$ glycerol/ $\mathrm{dH}_{2} \mathrm{O}$ on coverslips and visualized with an LSM 510 META inverted confocal microscope. Motoneuron length measurements of confocal pictures were done using the overlay function of the Zeiss LSM software with maximum projections of $\mathrm{z}$-stacks with constant stack height containing the whole neuronal projection. Images comparing 2 groups of fish were always done at the same time using identical settings. Paraffin sections were imaged on a Zeiss compound microscope. Images were assembled in Adobe Photoshop 8.0.
\end{abstract}

\section{Analysis of compound characteristics}

The GSK3 $\beta$ Kis were determined using a scintillation proximity assay with a biotinylated peptide sequence from eIF2B and $[\gamma-33 \mathrm{P}] \mathrm{ATP}$ as substrates. The CDK2 Kis were determined using a scintillation proximity assay with a biotinylated peptide sequence from GST retinoblastoma and [ $\gamma$-33P]ATP as substrates. Scintillation proximity assays and kinetic analyses were performed as described earlier (14). $\mathrm{IC}_{50}$ inhibition curves were analyzed by nonlinear regression using GraphPad Prism 5 (GraphPad Software).

$\mathrm{BBB}$ permeability in vitro was analyzed using an in vitro cell culture model for permeability across the BBB as described earlier (47).

\section{Analysis of GSK3 $\beta$ inhibition in TAU transfected cells}

3T3 fibroblasts were engineered to stably express 4-repeat TAU protein. The cells were treated with vehicle or with increasing concentrations of inhibitors and harvested at 4 hours after treatment. Cultures were then washed twice with $5 \mathrm{mM} \mathrm{MgCl} 2$ PBS. Extracts for WB analysis were prepared by homogenizing cells in ice-cold extraction buffer consisting of 20 mM HEPES, pH 7.4, 100 mM NaCl, 10 mM NaF, 1\% Triton X-100, 1 mM sodium orthovanadate, $10 \mathrm{mM}$ EDTA, and protease inhibitors $(2 \mathrm{mM}$ phenylmethylsulfonyl fluoride, $10 \mu \mathrm{g} / \mathrm{ml}$ aprotinin, $10 \mu \mathrm{g} / \mathrm{ml}$ leupeptin, and $10 \mu \mathrm{g} / \mathrm{ml}$ pepstatin). The samples were homogenized at $4^{\circ} \mathrm{C}$, and protein content was determined by the Bradford method. Total protein $(25 \mu \mathrm{g})$ was electrophoresed on $10 \%$ SDS-PAGE gel and transferred to a nitrocellulose membrane (Schleicher $\&$ Schuell Bioscience Inc.). The experiments were performed using the following primary antibodies: pS396, 1:1000, and Tau5, 1:1000. The filters were incubated with the antibody at $4{ }^{\circ} \mathrm{C}$ overnight in 5\% nonfat dried milk. A secondary horseradish peroxidaselinked sheep anti-mouse (1:1000; Amersham Biosciences) or horseradish peroxidase-linked donkey anti-rabbit (1:5000; Amersham Biosciences) followed by ECL detection reagents (Amersham Biosciences) was used for immunodetection. Quantitation of immunoreactivity was performed by densitometric scanning.

\section{Compound treatments}

Stock solutions were prepared by dissolving compounds in DMSO(SB-216763 and SB-415286, $20 \mathrm{mM}$ ), 50\% DMSO/ $\mathrm{dH}_{2} \mathrm{O}$ (AR-534, $5 \mathrm{mM}$ ), or $\mathrm{dH}_{2} \mathrm{O}$ (AR-164, $10 \mathrm{mM}$, and LiCl, $3 \mathrm{M}$ ). Stock solutions were diluted with embryo medium E3 containing 1\% DMSO to final dilutions of $50 \mu \mathrm{M}$ for SB-216763, $100 \mu \mathrm{M}$ for SB-415286, $100 \mathrm{mM}$ for LiCl, $25 \mu \mathrm{M}$ for AR-534, and $100 \mu \mathrm{M}$ for AR-164. The unpermeable chorions of fragile 4-hour-old embryos were only slightly opened to allow access to the compounds, while rather robust $24 \mathrm{hpf}$ embryos were completely dechorionated. Embryos were incubated in $1 \mathrm{ml}$ compound dilutions or control medium (E3 containing $1 \% \mathrm{DMSO}$ ) in 12-well plates; the solutions were changed daily. Embryos were either treated from 4 to $24 \mathrm{hpf}$ or from 24 to $100 \mathrm{hpf}$.

\section{Statistics}

Mean values and SD were calculated with Microsoft Excel, version 12.1.3. Statistical analysis was performed using 2-tailed Student's $t$ test in Microsoft Excel. Data are presented as mean \pm SD. $P<0.05$ was considered significant.

\section{Acknowledgments}

This work was supported by grants from the Deutsche Forschungsgemeinschaft (SFB 596 to B. Schmid and C. Haass; the Center for Integrated Protein Science to C. Haass and B. Schmid; and the Leibniz Award to C. Haass); Elitenetzwerk Bayern and Universität Bayern (to D. Paquet); the Federal Ministry for Education and Research (BMBF, BioFuture-Award 0311889 to R.W. Köster); the Studienstiftung des deutschen Volkes (to M. Distel); and the European Community's Seventh Framework Programme (FP7/2007-2013) under grant agreement no. 200611 (MEMOSAD to C. Haass, B. Schmid, E.-M. Mandelkow, and D. Paquet). C. Haass is supported by a research professorship at Ludwig-Maximilians-University. We thank A. Hruscha, M. Teucke, S. Schätzle, H. Kaiser, D. Drexler, O. Petrova, Y. Xu, E. Jerning, J. Neelissen, and Y. Nilsson for technical help; K. Kawakami, R. Rupp, and P. Lemaire for providing constructs; and P. Seubert, P. Davies, and C. Czech for providing antibodies. We thank the Hans and Ilse Breuer Foundation for the confocal microscope and $\mathrm{K}$. Winklhofer for critically reading this manuscript.

Received for publication September 23, 2008, and accepted in revised form February 25, 2009.

Address correspondence to: Christian Haass, Deutsches Zentrum für Neurodegenerative Erkrankungen (DZNE) and Adolf-Butenandt-Institut, Biochemistry, Ludwig-Maximilians-University, Schillerstr. 44, 80336 Munich, Germany. Phone: 49-89-2180-75472; Fax: 49-89-2180-75415; E-mail: chaass@med.uni-muenchen.de.
1. Haass, C., and Selkoe, D.J. 2007. Soluble protein oligomers in neurodegeneration: lessons from the Alzheimer's amyloid beta-peptide. Nat. Rev. Mol. Cell Biol. 8:101-112.

2. Kosik, K.S., Joachim, C.L., and Selkoe, D.J. 1986 Microtubule-associated protein tau (tau) is a major antigenic component of paired helical filaments in Alzheimer disease. Proc. Natl. Acad. Sci. U. S. A. 83:4044-4048.

3. Grundke-Iqbal, I., et al. 1986. Abnormal phosphorylation of the microtubule-associated protein tau (tau) in Alzheimer cytoskeletal pathology. Proc. Natl. Acad. Sci. U. S. A. 83:4913-4917.

4. Braak, H., and Braak, E. 1991. Neuropathological stageing of Alzheimer-related changes. Acta Neuropathol. 82:239-259.

5. Santacruz, K., et al. 2005. Tau suppression in a neurodegenerative mouse model improves memory function. Science. 309:476-481.

6. Hutton, M., et al. 1998. Association of missense and 5 '-splice-site mutations in tau with the inherited dementia FTDP-17. Nature. 393:702-705.

7. Mandelkow, E.M., and Mandelkow, E. 1998. Tau in Alzheimer's disease. Trends Cell Biol. 8:425-427.

8. Gotz, J., and Ittner, L.M. 2008. Animal models of Alzheimer's disease and frontotemporal dementia. Nat. Rev. Neurosci. 9:532-544.

9. Zon, L.I., and Peterson, R.T. 2005. In vivo drug discovery in the zebrafish. Nat. Rev. Drug Discov. 4:35-44

10. Jeong, J.Y., et al. 2008. Functional and developmental analysis of the blood-brain barrier in zebrafish. Brain Res. Bull. 75:619-628.

11. Tomasiewicz, H.G., Flaherty, D.B., Soria, J.P., and Wood, J.G. 2002. Transgenic zebrafish model of neurodegeneration. J. Neurosci. Res. 70:734-745.
12. Bai, Q., Garver, J.A., Hukriede, N.A., and Burton, E.A. 2007. Generation of a transgenic zebrafish model of tauopathy using a novel promoter element derived from the zebrafish eno2 gene. Nucleic Acids Res. 35:6501-6516.

13. Mazanetz, M.P., and Fischer, P.M. 2007. Untangling tau hyperphosphorylation in drug design for neurodegenerative diseases. Nat. Rev. Drug Discov. 6:464-479.

14. Bhat, R., et al. 2003. Structural insights and biological effects of glycogen synthase kinase 3-specific inhibitor AR-A014418. J. Biol. Chem. 278:45937-45945.

15. Winklhofer, K.F., Tatzelt, J., and Haass, C. 2008. The two faces of protein misfolding: gain- and lossof-function in neurodegenerative diseases. EMBOJ. 27:336-349.

16. Urasaki, A., Morvan, G., and Kawakami, K. 2006. 
Functional dissection of the Tol2 transposable element identified the minimal cis-sequence and a highly repetitive sequence in the subterminal region essential for transposition. Genetics. 174:639-649.

17. Koster, R.W., and Fraser, S.E. 2001. Tracing transgene expression in living zebrafish embryos. Dev. Biol. 233:329-346.

18. Walhout, A.J., et al. 2000. GATEWAY recombinational cloning: application to the cloning of large numbers of open reading frames or ORFeomes. Methods Enzymol. 328:575-592.

19. Park, H.C., et al. 2000. Analysis of upstream elements in the $\mathrm{HuC}$ promoter leads to the establishment of transgenic zebrafish with fluorescent neurons. Dev. Biol. 227:279-293.

20. Kosik, K.S., et al. 1988. Epitopes that span the tau molecule are shared with paired helical filaments. Neuron. 1:817-825.

21. Goedert, M., et al. 1994. Epitope mapping of monoclonal antibodies to the paired helical filaments of Alzheimer's disease: identification of phosphorylation sites in tau protein. Biochem. J. 301:871-877.

22. Seubert, P., et al. 1995. Detection of phosphorylated Ser262 in fetal tau, adult tau, and paired helical filament tau. J. Biol. Chem. 270:18917-18922.

23. Greenberg, S.G., Davies, P., Schein, J.D., and Binder, L.I. 1992. Hydrofluoric acid-treated tau PHF proteins display the same biochemical properties as normal tau. J. Biol. Chem. 267:564-569.

24. Hasegawa, M., et al. 1996. Characterization of mAb AP422, a novel phosphorylation-dependent monoclonal antibody against tau protein. FEBS Lett. 384:25-30.

25. Biernat, J., et al. 1992. The switch of tau protein to an Alzheimer-like state includes the phosphorylation of two serine-proline motifs upstream of the microtubule binding region. ЕМВO J. 11:1593-1597.

26. Jicha, G.A., Berenfeld, B., and Davies, P. 1999.
Sequence requirements for formation of conformational variants of tau similar to those found in Alzheimer's disease. J. Neurosci. Res. 55:713-723.

27. Furutani-Seiki, M., et al. 1996. Neural degeneration mutants in the zebrafish, Danio rerio. Development. 123:229-239.

28. Thies, E., and Mandelkow, E.M. 2007. Missorting of tau in neurons causes degeneration of synapses that can be rescued by the kinase MARK2/Par-1. J. Neurosci. 27:2896-2907.

29. Terwel, D., et al. 2005. Changed conformation of mutant Tau-P301L underlies the moribund tauopathy, absent in progressive, nonlethal axonopathy of Tau-4R/2N transgenic mice. J. Biol. Chem. 280:3963-3973.

30. Trevarrow, B., Marks, D.L., and Kimmel, C.B. 1990 Organization of hindbrain segments in the zebrafish embryo. Neuron. 4:669-679.

31. Fox, M.A., and Sanes, J.R. 2007. Synaptotagmin I and II are present in distinct subsets of central synapses. J. Comp. Neurol. 503:280-296.

32. Myers, P.Z., Eisen, J.S., and Westerfield, M. 1986. Development and axonal outgrowth of identified motoneurons in the zebrafish. J. Neurosci. 6:2278-2289.

33. Smith, D.G., et al. 2001. 3-Anilino-4-arylmaleimides: potent and selective inhibitors of glycogen synthase kinase-3 (GSK-3). Bioorg. Med. Chem. Lett. 11:635-639.

34. Hong, M., Chen, D.C., Klein, P.S., and Lee, V.M. 1997. Lithium reduces tau phosphorylation by inhibition of glycogen synthase kinase-3. J. Biol. Chem. 272:25326-25332.

35. Doble, B.W., and Woodgett, J.R. 2003. GSK-3: tricks of the trade for a multi-tasking kinase. J. Cell Sci. 116:1175-1186.

36. Stachel, S.E., Grunwald, D.J., and Myers, P.Z. 1993. Lithium perturbation and goosecoid expression identify a dorsal specification pathway in the pre- gastrula zebrafish. Development. 117:1261-1274.

37. Geling, A., Steiner, H., Willem, M., Bally-Cuif, L., and Haass, C. 2002. A gamma-secretase inhibitor blocks Notch signaling in vivo and causes a severe neurogenic phenotype in zebrafish. EMBO Rep. 3:688-694.

38. Gerhard, G.S., et al. 2002. Life spans and senescent phenotypes in two strains of Zebrafish (Danio rerio). Exp. Gerontol. 37:1055-1068.

39. Roberts, R.C. 1961. The lifetime growth and reproduction of selected strains of mice. Heredity. 16:369-381.

40. Koster, R.W., and Fraser, S.E. 2001. Direct imaging of in vivo neuronal migration in the developing cerebellum. Curr. Biol. 11:1858-1863.

41. Mullins, M.C., Hammerschmidt, M., Haffter, P., and Nusslein-Volhard, C. 1994. Large-scale mutagenesis in the zebrafish: in search of genes controlling development in a vertebrate. Curr. Biol. 4:189-202.

42. Kimmel, C.B., Ballard, W.W., Kimmel, S.R., Ullmann, B., and Schilling, T.F. 1995. Stages of embryonic development of the zebrafish. Dev. Dyn. 203:253-310.

43. Bevis, B.J., and Glick, B.S. 2002. Rapidly maturing variants of the Discosoma red fluorescent protein (DsRed). Nat. Biotechnol. 20:83-87.

44. Kawakami, K. 2005. Transposon tools and methods in zebrafish. Dev. Dyn. 234:244-254.

45. Nüsslein-Volhard, C., and Dahm, R. 2002. Zebrafish: a practical approach. Oxford University Press. Oxford, United Kingdom. 303 pp.

46. Mocanu, M.M., et al. 2008. The potential for betastructure in the repeat domain of tau protein determines aggregation, synaptic decay, neuronal loss, and coassembly with endogenous Tau in inducible mouse models of tauopathy. J. Neurosci. 28:737-748.

47. Cecchelli, R., et al. 1999. In vitro model for evaluating drug transport across the blood-brain barrier. Adv. Drug Deliv. Rev. 36:165-178. 